Review

\title{
Assessing the human health impacts of exposure to disinfection by-products - A critical review of concepts and methods
}

\author{
James Grellier a,b,c,d,*, Lesley Rushton ${ }^{\mathrm{d}}$, David J. Briggs ${ }^{\mathrm{d}}$, Mark J. Nieuwenhuijsen ${ }^{\mathrm{a}, \mathrm{b}, \mathrm{c}, \mathrm{d}}$ \\ a Centre for Research in Environmental Epidemiology (CREAL), Barcelona, Spain \\ b Universitat Pompeu Fabra (UPF), Barcelona, Spain \\ c CIBER Epidemiología y Salud Pública (CIBERESP), Madrid, Spain \\ ${ }^{d}$ Department of Epidemiology and Biostatistics, School of Public Health, Faculty of Medicine, Imperial College, London, United Kingdom
}

\section{A R T I C L E I N F O}

\section{Article history:}

Received 9 July 2014

Received in revised form 28 January 2015

Accepted 1 February 2015

Available online 11 March 2015

\section{Keywords:}

Risk assessment

Disinfection by-products

Chloroform

Health impact assessment

Health risks

Cancer

\begin{abstract}
A B S T R A C T
Understanding the public health implications of chemical contamination of drinking water is important for societies and their decision-makers. The possible population health impacts associated with exposure to disinfection by-products (DBPs) are of particular interest due to their potential carcinogenicity and their widespread occurrence as a result of treatments employed to control waterborne infectious disease.

We searched the literature for studies that have attempted quantitatively to assess population health impacts and health risks associated with exposure to DBPs in drinking water. We summarised and evaluated these assessments in terms of their objectives, methods, treatment of uncertainties, and interpretation and communication of results.

In total we identified 40 studies matching our search criteria. The vast majority of studies presented estimates of generic cancer and non-cancer risks based on toxicological data and methods that were designed with regulatory, health-protective purposes in mind, and therefore presented imprecise and biased estimates of health impacts. Many studies insufficiently addressed the numerous challenges to DBP risk assessment, failing to evaluate the evidence for a causal relationship, not appropriately addressing the complex nature of DBP occurrence as a mixture of chemicals, not adequately characterising exposure in space and time, not defining specific health outcomes, not accounting for characteristics of target populations, and not balancing potential risks of DBPs against the health benefits related with drinking water disinfection. Uncertainties were often poorly explained or insufficiently accounted for, and important limitations of data and methods frequently not discussed. Grave conceptual and methodological limitations in study design, as well as erroneous use of available dose-response data, seriously impede the extent to which many of these assessments contribute to understanding the public health implications of exposure to DBPs. In some cases, assessment results may cause unwarranted alarm among the public and potentially lead to poor decisions being made in sourcing, treatment, and provision of drinking water. We recommend that the assessment of public health impacts of DBPs should be viewed as a means of answering real world policy questions relating to drinking water quality, including microbial contaminants; that they should be conducted using the most appropriate and up-to-date data and methods, and that associated uncertainties and limitations should be accounted for using quantitative methods where appropriate.
\end{abstract}

(c) 2015 Elsevier Ltd. All rights reserved.

\section{Contents}

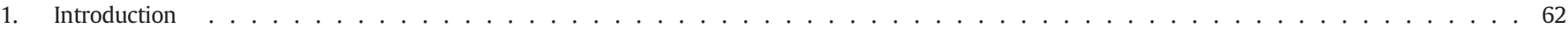

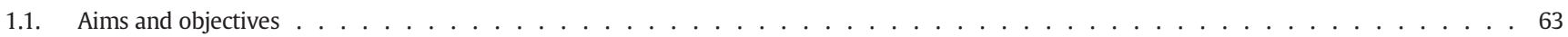

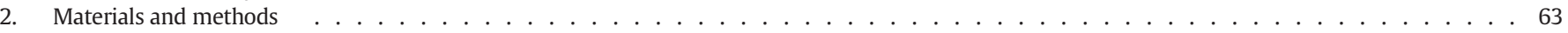

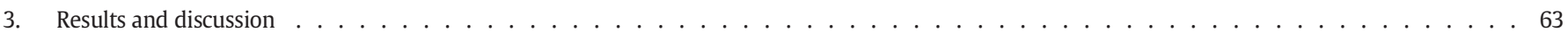

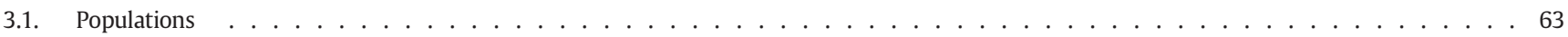

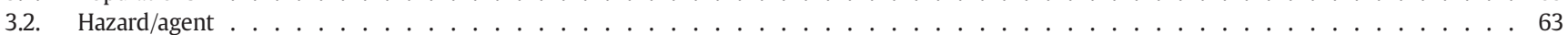

\footnotetext{
Funding: This research was supported by the Integrated Assessment of Health Risks of Environmental Stressors in Europe (INTARESE) project, which was funded by the European Commission Sixth Framework Programme (2002-2006) grant GOCE-CT-2005-018385.

* Corresponding author at: CREAL - Centre for Research in Environmental Epidemiology, Parc de Recerca Biomèdica de Barcelona (despatx 183.15), Doctor Aiguader, 88, 08003 Barcelona, Spain.

E-mail address: jgrellier@creal.cat (J. Grellier).
} 


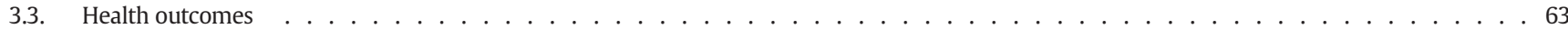

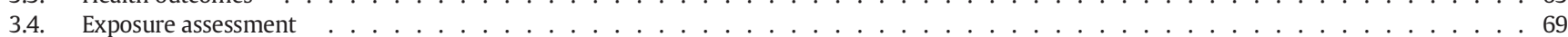

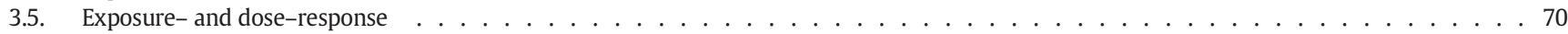

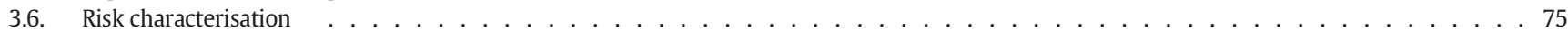

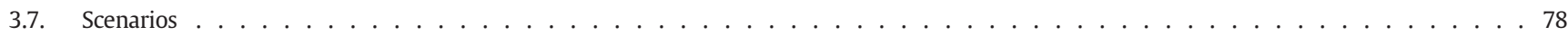

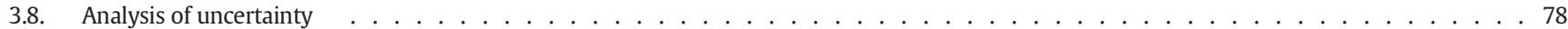

3.9. Interpretation and presentation of assessment results - risk communication $\ldots \ldots \ldots$

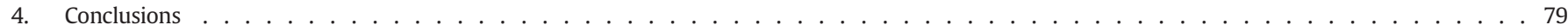

Appendix A. Supplementary data . . . . . . . . . . . . . . . . . . . . . . . . . . . . . . . . . . . . .

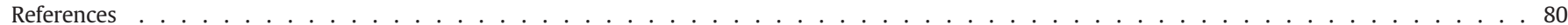

\section{Introduction}

Maintaining high microbial quality of drinking water is a cornerstone of public health, and chemical disinfection with chlorine and its compounds commonly forms a key component in drinking water treatment. Disinfection by-products (DBPs) may result from reactions between disinfectants and impurities in source water and their occurrence has been a public health concern for the last four decades.

DBPs were first identified in the form of trihalomethanes (THMs) (Bellar et al., 1974; Rook, 1974). By concentration, THMs represent the most prevalent group of DBPs in typical chlorinated drinking water, but many hundreds of other DBPs also occur (Nieuwenhuijsen et al., 2000a; WHO, 2006), several of which are potentially carcinogenic (Richardson et al., 2007). The $\sim 600$ DBPs that have been identified (Richardson, 1998; Richardson et al., 2007) represent only a small fraction of the total organic halides present in chlorinated supplies (USEPA, 1998), and relatively few of those chemicals have been adequately characterised in terms of occurrence. Fewer still have been assessed in terms of their potential effects on human health (Richardson et al., 2007).

The occurrence of DBPs has become an issue of interest to policy makers, drinking water providers and engineers, epidemiologists, biologists and risk assessors, particularly since they are a side effect of disinfection with chlorine, which commonly plays an important role in minimising public health risks of waterborne infectious diseases. The concentrations of DBPs vary temporally and geographically according to the physicochemical properties of source water, the nature of treatment and distribution systems, and climate (Amy et al., 2000; Nieuwenhuijsen et al., 2000b), which makes characterisation of general population exposures for the purposes of epidemiological studies and population health impact assessments complex.

Epidemiological studies based on routine monitoring data (e.g. THM concentrations measured at individual residences as a proxy for an unknown putative agent in the DBP mixture) have been carried out for several cancers and several non-cancer health endpoints, in particular birth outcomes (Nieuwenhuijsen et al., 2013). Various systematic reviews, meta-analyses and pooled analyses of cancer studies have also been produced (Amy et al., 2000; Cantor, 1997; Morris et al., 1992; Nieuwenhuijsen et al., 2009a; Villanueva et al., 2003), these show no consistent evidence of associations between DBP exposure and the majority of cancers (Amy et al., 2000). Consistent, positive associations have been found only for bladder cancer: meta- and pooled analyses have demonstrated exposure-response relationships between average residential THM concentrations and bladder cancer in men (Costet et al., 2011; Villanueva et al., 2003, 2004). In terms of non-cancer outcomes, small positive associations have been reported for still births (Nieuwenhuijsen et al., 2010), small for gestational age (Grellier et al., 2010) and congenital anomalies (Nieuwenhuijsen et al., 2009b). For all of these health outcomes, epidemiological evidence is currently not sufficient to infer whether such associations are causal.

Regulatory limits for DBPs have been developed chiefly from data derived from animal toxicological studies. EU guidelines specify a parametric value of $100 \mu \mathrm{g} / \mathrm{L}$ for the four most prevalent THMs (THM4) (European Commission, 1998); the maximum contaminant level in the US is currently $80 \mu \mathrm{g} / \mathrm{L}$ for THM4 (USEPA, 1998). Five haloacetic acids are also regulated in the US, as well as bromate and chlorite (USEPA, 2013). In each case, limits have been set according to available scientific knowledge and account was taken of the precautionary principle, such that life-long consumption of water meeting the guideline values should afford the public protection from adverse health effects. However, cytotoxicological and genotoxicological studies of currently unregulated DBPs indicate that many may be considerably more toxic to humans than THMs or HAAs (Plewa and Wagner, 2009; Plewa et al., 2004, 2008; Richardson et al., 2007). Although THMs make up a high proportion of DBPs in chlorinated drinking water by concentration, it is plausible that, as more data become available on the occurrence and carcinogenicity of some of these unregulated DBPs, the relevance of THMs as a carcinogenic risk factor will diminish considerably (Bull, 2012).

Risk assessment carried out with the aim of determining regulatory limits involves extensive review of both epidemiological and toxicological data. Animal studies tend to be relied upon for providing quantitative dose-response data. Measures of cancer potency-referred to as either potency factors (PF) or slope factors (SF)-are derived by modelling animal carcinogenicity data for each applicable exposure pathway (ingestion, inhalation and dermal absorption) for the most sensitive cancer endpoint. For a suspected genotoxic carcinogen, it is assumed that exposure at any level increases the probability of cancer. The linearised multistage (LMS) model (Crump, 1984) has been used extensively as the default means of extrapolating from the high doses used in animal studies to the lower doses typically experienced by humans. Usually, cancer potency factors are defined using the 95\% upper bound of the LMS regression line so as to allow for uncertainties resulting from extrapolation from animals to humans, and from differences in the exposure regimes. In order to calculate regulatory limits, lifetime exposure of a typical human to a potential carcinogen in drinking water is then estimated via each applicable exposure pathway (drinking, washing, showering etc.). An upper bound estimate of lifetime excess cancer risk (LECR)-the lifetime probability of a typical individual developing a cancer-is then estimated by summing the products of exposure through each route by its appropriate potency factor, according to the procedure described by Chrostowski (1994), Gratt (1996) and USEPA (2002a):

$\mathrm{LECR}=\sum \mathrm{LAE}_{\mathrm{i}} \times \mathrm{PF}_{\mathrm{i}}$

where LAE is the lifetime average exposure $(\mathrm{mg}), \mathrm{PF}$ is the potency factor $\left((\mathrm{mg} / \mathrm{kg} \text {-day })^{-1}\right)$, and i is the exposure route (ingestion, inhalation and absorption). Since the LECR is based on potency factors derived from the upper bound of the LMS model, it can be considered a conservative overestimate that should be protective of public health. Regulatory guidelines use this information to set a concentration of a chemical in drinking water at which the lifetime risk of cancer is considered acceptable (commonly between $1 \times 10^{-4}$ and $1 \times 10^{-6}$, depending on circumstances). 
Non-carcinogenic health risk is quantified as a hazard quotient (HQ), calculated from the same estimate of LAE divided by a reference dose (RfD), which is a measure of the potential of a systemic toxic effect in a lifetime of exposure, again commonly derived from animal data. In contrast to genotoxic carcinogenic effects, systemic effects are considered to occur only above an identifiable threshold of exposure at which the body's capacity to detoxify an agent through homeostasis and adaptive mechanisms is exceeded. In order to derive an RfD from animal data, it is necessary, firstly, to identify the no observed adverse effect level (NOAEL), the dose at which no biologically or statistically significant effects are observed in study animals. To estimate a level at which human health is protected, the NOAEL is then divided by a number of uncertainty factors (each typically equal to 10), to account for: (a) variation in sensitivity between among members of the human population; (b) uncertainty involved in extrapolating from animal data to humans; and (c) for extrapolation from subchronic assays to chronic exposure conditions. The RfD can thus be considered the threshold of exposure at which there is no appreciable risk of deleterious systemic effects over a lifetime. Hence, an HQ of less than unity indicates that an individual should not experience adverse non-carcinogenic health effects.

Outside of the regulatory sphere, there are other decision-making contexts in which it is useful to estimate the health impacts associated with exposure to environmental exposures as accurately as possible, without employing conservative, public health-protective assumptions. For the purposes of this review, we broadly refer to such efforts as population health impact assessments, although many other terms have been used to describe such studies (Briggs, 2008). Over the last few decades, several studies have been carried out with population health impact assessment as an aim, but which utilise methods and data developed for regulatory risk assessment. Conspicuously fewer population health impact assessments have been done using epidemiological data, or for the purposes of looking at benefits of disinfection versus possible health risks associated with resultant DBPs.

\subsection{Aims and objectives}

We sought to identify and describe published studies that aim to estimate population health impacts related to exposure to DBPs, with a view to assessing how well this objective has been met in the literature to date. Our specific objectives were to identify the methods used to quantify health risks and impacts, to review the means by which various types of uncertainty were accounted for, and to discuss the ways in which results of such assessments have been interpreted, contextualised, and presented (risk communication). We aimed to provide recommendations for the improvement of population health impact assessment related to exposure to DBPs, and to environmental stressors more generally.

\section{Materials and methods}

A literature search was carried out to identify studies claiming to assess population health impacts of exposure to DBPs in drinking water (search method presented in Supplementary material 1). Studies were considered eligible if they presented quantitative predictions of health impact or health risk associated with exposure to DBPs for an identified human population. We reviewed all identified studies in detail and their main features were summarised, focusing in particular on hazard/agent, health outcomes, exposure assessment, target populations, risk characterisation, temporal character, scenarios or policy questions analysed, analysis of uncertainties, and interpretation and presentation of the assessment results. Detailed review of the studies allowed us to evaluate the strengths and shortcomings of methods typically used, and to make recommendations on how assessment of population health impacts of exposure to DBPs might be improved.

\section{Results and discussion}

In total, we identified 40 population health impact assessment studies that met our eligibility criteria for inclusion in this review (Table 1).

\subsection{Populations}

The populations defined in the majority of studies were those of individual countries, regions, cities or those served by individual waterworks or distribution systems. Eight studies were done in Canada, 8 in the USA, 5 in Turkey, 3 in China (one jointly reported with a Canadian study), 2 in Korea, 2 in Pakistan, 2 in Taiwan, and one each in Brazil, Germany, Hong Kong, India, Italy, Japan, Lebanon, Mexico, the Netherlands and New Zealand. Hypothetical populations were defined in 2 studies (Black et al., 1996; Havelaar et al., 2000), and one laboratory-based study focused on 6 subjects exposed to DBPs under experimental conditions (Jo et al., 1990). In one study, sensitive population subgroups were identified, comprising women of reproductive age and developing foetuses (USEPA, 2005). Surveyed demographic characteristics pertaining to these populations (in terms of sex- or agestructure) were taken into account in few assessments, and commonly existing general point estimates or distributions of body weight, water consumption rates, inhalation rates etc. were used when characterising exposures. In some cases not taking into account the demographic structure or behavioural characteristics of populations may result in a misclassification of the risk status of certain population strata. For example, bladder cancer has been found to be associated with DBPs only in males, and incident cases occur extremely infrequently in those below the age of 40 years. Estimation of numbers of cases attributable to exposure to DBPs should be made with adequate consideration of both age and sex stratification of the target population.

\subsection{Hazard/agent}

Twenty-four studies investigated solely THM4, 8 studies focused only on trichloromethane (TCM), and 5 studies looked at THM4 and some HAAs. One study focused on TCM, bromodichloromethane (BDCM) and dibromochloromethane (DBCM) only. One study looked at ingestion of bromate, which is produced due to disinfection with ozone, and one used residence in a zone where drinking water was chlorinated as an indicator of DBP exposure. Only one study explicitly considered contamination of drinking water by pathogens (specifically Cryptosporidium parvum), in spite of the very clear policy need for assessments in which cancer risks associated with DBPs are balanced with the benefits of disinfection (Havelaar et al., 2000).

\subsection{Health outcomes}

Almost all studies focused on the risk of cancer, with the vast majority of studies estimating LECR rather than risks of specific cancers. This method requires the use of PFs that are derived from the most sensitive tumour endpoint in a set of animal studies. Although theoretically this is protective against other cancers, it cannot be assumed that this same endpoint would be that occurring in humans. As such, the degree to which the LECR calculated provides an upper bound estimate of risk of any cancer is unknown. Five studies looked at specific cancers: bladder cancer (Attias et al., 1995; Malcolm et al., 1999; USEPA, 2005), colorectal cancer (Malcolm et al., 1999; USEPA, 2005), and renal cell cancer (Havelaar et al., 2000). Generic non-carcinogenic risks were estimated in 14 studies, quantified using hazard quotients (or hazard indices). Specific non-cancer outcomes included microbial infections (Havelaar et al., 2000), foetal loss (USEPA, 2005), and birth defects (Malcolm et al., 1999).

Metrics such as LECR and HI are not particularly informative in population health impact assessment. Upper bound estimates of LECR 
Table 1

Summary of health risk and impact assessments of drinking water disinfection by-products.

\begin{tabular}{|c|c|c|c|c|c|c|c|c|c|c|c|}
\hline \multirow[b]{2}{*}{ Reference } & \multicolumn{5}{|c|}{ Issue framing } & \multicolumn{2}{|c|}{ Exposure assessment } & \multirow[b]{2}{*}{$\begin{array}{c}\text { Exposure- } \\
\text { /dose-response }\end{array}$} & \multirow[b]{2}{*}{$\begin{array}{c}\text { Risk } \\
\text { characterisation }\end{array}$} & \multirow[b]{2}{*}{$\begin{array}{l}\text { Analysis of } \\
\text { uncertainty }\end{array}$} & \multirow[b]{2}{*}{$\begin{array}{l}\text { Outcome } \\
\text { metric }\end{array}$} \\
\hline & $\begin{array}{c}\text { Target } \\
\text { population }\end{array}$ & $\begin{array}{c}\text { Hazard/ } \\
\text { agent }\end{array}$ & Health outcome & $\begin{array}{l}\text { Temporal } \\
\text { framing }\end{array}$ & $\begin{array}{l}\text { Scenario(s) } \\
\text { considered }\end{array}$ & $\begin{array}{l}\text { Exposure } \\
\text { route }\end{array}$ & $\begin{array}{l}\text { Exposure } \\
\text { assessment } \\
\text { method }\end{array}$ & & & & \\
\hline $\begin{array}{l}\text { (Lahey \& } \\
\text { Connor 1983) }\end{array}$ & $\begin{array}{l}\text { Population } \\
\text { of Nassau } \\
\text { County, } \\
\text { Long Island, } \\
\text { USA }\end{array}$ & $\begin{array}{l}\text { Chlorinated } \\
\text { chemicals } \\
\text { including } \\
\text { TCM - } \\
\text { unclear if } \\
\text { TCM is as } \\
\text { DBP or other } \\
\text { source in } \\
\text { ground water }\end{array}$ & $\begin{array}{l}\text { Cancer } \\
\text { (unspecified) }\end{array}$ & $\begin{array}{l}\text { Not } \\
\text { specified }\end{array}$ & $\begin{array}{l}\text { Disposal of } \\
\text { chemical waste } \\
\text { on land and at } \\
\text { sea }\end{array}$ & $\begin{array}{l}\text { Ingestion of } \\
\text { DW (and } \\
\text { ingestion of } \\
\text { fish) }\end{array}$ & \begin{tabular}{|l} 
Doses \\
estimated by \\
assuming 2 \\
$1 /$ day (and $6.5 \mathrm{~g}$ \\
fish/ day) \\
\\
No details of \\
levels of TCM \\
in water \\
presented
\end{tabular} & $\begin{array}{l}\text { Upper bound } \\
\text { PF (source of } \\
\text { data not } \\
\text { presented) }\end{array}$ & $\begin{array}{l}\text { LECR estimated } \\
\text { as product of } \\
\text { potency by } \\
\text { estimated dose } \\
\text { (few details } \\
\text { provided) } \\
\text { Sum of risks for } \\
\text { each chlorinated } \\
\text { chemical }\end{array}$ & $\mathrm{N} / \mathrm{a}$ & $\begin{array}{l}\text { LECR for } \\
\text { entire } \\
\text { population } \\
\text { due to } \\
\text { ingestion of } \\
\text { TCM }\end{array}$ \\
\hline (Jo et al. 1990) & $\begin{array}{l}\text { Six subjects } \\
\text { in an } \\
\text { experimental } \\
\text { study, New } \\
\text { Jersey, USA }\end{array}$ & $\mathrm{TCM}$ & $\begin{array}{l}\text { Cancer } \\
\text { (unspecified) }\end{array}$ & $\begin{array}{l}\text { Water } \\
\text { samples } \\
\text { taken prior } \\
\text { to } \\
\text { showering-- } \\
\text { risks } \\
\text { estimated } \\
\text { over a } \\
\text { lifetime }\end{array}$ & $\begin{array}{l}\text { Comparison } \\
\text { between risks } \\
\text { associated with } \\
\text { drinking or } \\
\text { showering in } \\
\text { the same DW }\end{array}$ & $\begin{array}{l}\text { Multipathway } \\
\text { (ingestion } \\
\text { of DW, } \\
\text { dermal } \\
\text { contact and } \\
\text { inhalation via } \\
\text { showering) }\end{array}$ & $\begin{array}{l}\begin{array}{l}\text { Tap water } \\
\text { sampled after } \\
\text { each of } 13 \\
\text { showers }\end{array} \\
\text { Shower dose: } \\
\text { breath analysis } \\
\text { for TCM pre- } \\
\text { and post- } \\
\text { showering }\end{array}$ & $\begin{array}{l}\text { Upper bound } \\
\text { PF derived } \\
\text { from linearised } \\
\text { model of single } \\
\text { ingestion } \\
\text { animal study } \\
\text { Ingestion PF } \\
\text { applied to all } \\
\text { exposure routes }\end{array}$ & $\begin{array}{l}\text { Doses were } \\
\text { established with } \\
\text { deterministic } \\
\text { models, using } \\
\text { general } \\
\text { population } \\
\text { exposure } \\
\text { parameters } \\
\\
\text { LECR calculated } \\
\text { from dose and PF }\end{array}$ & $\mathrm{N} / \mathrm{a}$ & $\begin{array}{l}\text { LECC due to } \\
\text { dose of TCM } \\
\text { in a } \\
\text { theoretical } \\
\text { population of } \\
\text { one million }\end{array}$ \\
\hline $\begin{array}{l}\text { (Attias et al. } \\
1995 \text { ) }\end{array}$ & $\begin{array}{l}\text { Five regions } \\
\text { of Sardinia, } \\
\text { Italy }\end{array}$ & THM4 & $\begin{array}{l}\text { Cancer } \\
\text { (unspecified) } \\
\text { using } \\
\text { toxicological } \\
\text { data, and } \\
\text { bladder cancer } \\
\text { using } \\
\text { epidemiologic } \\
\text { data }\end{array}$ & $\begin{array}{l}\text { THM4 } \\
\text { levels } \\
\text { assumed to } \\
\text { be constant } \\
\text { over } \\
\text { previous } \\
2 \text { decades. } \\
\text { Baseline } \\
\text { rates of } \\
\text { bladder } \\
\text { cancer } \\
\text { averaged } \\
\text { over 2 } \\
\text { decades } \\
\text { (latency) }\end{array}$ & $\begin{array}{l}\text { Comparison of } \\
\text { estimations of } \\
\text { response using } \\
\text { toxicological } \\
\text { and } \\
\text { epidemiologic } \\
\text { methods }\end{array}$ & $\begin{array}{l}\text { Ingestion of } \\
\text { DW }\end{array}$ & $\begin{array}{l}\text { Household DW } \\
\text { monitoring data }\end{array}$ & $\begin{array}{l}\text { PFs estimated } \\
\text { from } \\
\text { toxicological } \\
\text { data using both } \\
\text { multistage and } \\
\text { linearised } \\
\text { multistage } \\
\text { models } \\
\text { Epidemiologic } \\
\text { risk estimates } \\
\text { adapted from a } \\
\text { meta-analysis } \\
\text { on bladder } \\
\text { cancer (Morris } \\
\text { et al. 1992) }\end{array}$ & $\begin{array}{l}\text { For animal data: } \\
\text { unit risks } \\
\text { From human data: } \\
\text { unit lifetime risk } \\
\text { expressed as } \\
\text { incremental } \\
\text { lifetime } \\
\text { carcinogenic risk } \\
\text { (derived from } \\
\text { average relative } \\
\text { risk model, } \\
\text { incorporating } \\
\text { baseline rates of } \\
\text { bladder cancer) }\end{array}$ & $\begin{array}{l}\text { Analysis of } \\
\text { uncertainties } \\
\text { introduced due to } \\
\text { using different } \\
\text { data and methods } \\
\text { (toxicological/ } \\
\text { epidemiological) } \\
\text { plus presentation } \\
\text { of risk estimates } \\
\text { based on MLE PF } \\
\text { (in addition to } \\
\text { upper bounds) }\end{array}$ & $\begin{array}{l}\text { Unit risks } \\
\text { (incremental } \\
\text { lifetime } \\
\text { carcinogenic } \\
\text { risk per } \mu \mathrm{g} / \mathrm{l} \\
\text { of THM) } \\
\text { expressed as } \\
\text { the } \\
\text { probability of } \\
\text { tumour } \\
\text { induction }\end{array}$ \\
\hline $\begin{array}{l}\text { (Black et al. } \\
1996 \text { ) }\end{array}$ & $\begin{array}{l}\text { Hypothetical } \\
\text { population } \\
\text { served by a } \\
\text { typical } \\
\text { surface } \\
\text { water } \\
\text { DWTP in } \\
\text { the USA }\end{array}$ & $\begin{array}{l}\text { THM4 and } 2 \\
\text { HAAs (no risk } \\
\text { analysis } \\
\text { presented for } \\
\text { HAAs) }\end{array}$ & $\begin{array}{l}\text { Cancer } \\
\text { (unspecified) }\end{array}$ & $\begin{array}{l}\text { Simulated } \\
\text { exposure } \\
\text { annual risk } \\
\text { calculated }\end{array}$ & $\begin{array}{l}\text { Investigation } \\
\text { of the effects } \\
\text { on health risks } \\
\text { under a } \\
\text { number of } \\
\text { scenarios } \\
\text { relating to } \\
\text { implementation } \\
\text { of organic } \\
\text { carbon } \\
\text { removal } \\
\text { technologies }\end{array}$ & $\begin{array}{l}\text { Ingestion of } \\
\text { DW }\end{array}$ & $\begin{array}{l}\text { Exposure } \\
\text { entirely } \\
\text { simulated: } \\
\text { formation of } \\
\text { THM4 at the } \\
\text { DWTP due to } \\
\text { treatment of } \\
\text { typical US } \\
\text { surface water } \\
\text { was modelled } \\
\text { using a number } \\
\text { of predictive } \\
\text { models }\end{array}$ & $\begin{array}{l}\text { Published upper } \\
\text { bound } \mathrm{PF}_{\text {ingestion }}\end{array}$ & $\begin{array}{l}\text { Risk estimates } \\
\text { calculated as } \\
\text { product of PFs } \\
\text { and modelled } \\
\text { exposures }\end{array}$ & $\begin{array}{l}\text { Sensitivity } \\
\text { analysis of } \\
\text { parameters in } \\
\text { treatment } \\
\text { technologies } \\
\text { An extensive list } \\
\text { of modelling } \\
\text { assumptions is } \\
\text { provided }\end{array}$ & $\begin{array}{l}\text { Annual cases } \\
\text { of cancer } \\
\text { attributable to } \\
\text { exposures in } \\
\text { a theoretical } \\
\text { population of } \\
\text { one million }\end{array}$ \\
\hline $\begin{array}{l}\text { (Chung et al. } \\
1997 \text { ) }\end{array}$ & $\begin{array}{l}\text { Six major } \\
\text { river } \\
\text { systems and } \\
\text { their } \\
\text { distribution } \\
\text { systems } \\
\text { across the } \\
\text { Republic of } \\
\text { Korea }\end{array}$ & $\begin{array}{l}\text { THMs (as part } \\
\text { of a suite of } \\
80 \\
\text { contaminants } \\
\text { including } \\
\text { VOCs, PAHs, } \\
\text { pesticides, } \\
\text { metals) }\end{array}$ & $\begin{array}{l}\text { Cancer } \\
\text { (unspecified) }\end{array}$ & $\begin{array}{l}\text { Two years } \\
\text { (1993- } \\
\text { 1995) of } \\
\text { exposure } \\
\text { estimates } \\
\text { extrapolated } \\
\text { over } \\
\text { lifetime }\end{array}$ & $\begin{array}{l}\text { Comparison of } \\
\text { risks } \\
\text { (calculated } \\
\text { against an } \\
\text { implicit zero } \\
\text { risk baseline) } \\
\text { for each } \\
\text { contaminant as } \\
\text { means of } \\
\text { prioritising } \\
\text { DW } \\
\text { monitoring }\end{array}$ & $\begin{array}{l}\text { Multipathway } \\
\text { (ingestion } \\
\text { of DW, } \\
\text { dermal } \\
\text { contact and } \\
\text { inhalation via } \\
\text { showering) }\end{array}$ & $\begin{array}{l}\text { LADE } \\
\text { estimated using } \\
\text { PDFs of body } \\
\text { weight and } \\
\text { consumption, } \\
\text { based on } \\
\text { sampling of } \\
\text { raw, treated and } \\
\text { tap water in } 6 \\
\text { cities }\end{array}$ & $\begin{array}{l}\text { Upper bound } \\
\text { PFs (published } \\
\text { and derived } \\
\text { from animal } \\
\text { data) }\end{array}$ & $\begin{array}{l}\text { For carcinogens: } \\
\text { LADE multiplied } \\
\text { by upper } 95 \% \text { CI } \\
\text { on PFs derived } \\
\text { using multistage } \\
\text { model } \\
\text { (No THMs were } \\
\text { considered as } \\
\text { non-carcinogens) }\end{array}$ & MCS (exposure) & $\begin{array}{l}\text { Lifetime } \\
\text { excess cancer } \\
\text { risks } \\
\text { attributable to } \\
\text { exposure to } \\
\text { THMs }\end{array}$ \\
\hline $\begin{array}{l}\text { (Malcolm et al. } \\
\text { 1999) }\end{array}$ & $\begin{array}{l}\text { New } \\
\text { Zealand }\end{array}$ & $\begin{array}{l}\text { Chlorination } \\
\text { DBPs }\end{array}$ & $\begin{array}{l}\text { Cancers of the } \\
\text { bladder, rectum } \\
\text { and colon; birth } \\
\text { defects }\end{array}$ & Annual & $\begin{array}{l}\text { Disinfection vs } \\
\text { baseline } \\
\text { scenario of no } \\
\text { disinfection } \\
\text { (chemical risk } \\
\text { factors only) }\end{array}$ & $\begin{array}{l}\text { Ingestion of } \\
\text { DW (not } \\
\text { specified) }\end{array}$ & $\begin{array}{l}\text { Rough } \\
\text { approximations } \\
\text { of ever-never } \\
\text { exposure to } \\
\text { chlorinated } \\
\text { water }\end{array}$ & $\begin{array}{l}\text { Relative risk } \\
\text { from } \\
\text { epidemiological } \\
\text { study }\end{array}$ & $\begin{array}{l}\text { PAR\% for all } \\
\text { health outcomes; } \\
\text { then applied to } \\
\text { cancer and birth } \\
\text { defect registry } \\
\text { data }\end{array}$ & N/a & $\begin{array}{l}\text { Annual cases } \\
\text { of bladder, } \\
\text { rectum and } \\
\text { colon cancer, } \\
\text { and cases of } \\
\text { birth defects, } \\
\text { attributable to } \\
\text { exposure to } \\
\text { DBPs }\end{array}$ \\
\hline $\begin{array}{l}\text { (Havelaar et al. } \\
2000 \text { ) }\end{array}$ & $\begin{array}{l}\text { Hypothetical } \\
\text { population } \\
\text { served by an } \\
\text { ozonating } \\
\text { DWTP in } \\
\text { the } \\
\text { Netherlands } \\
\text { (including } \\
\text { immune- } \\
\text { compromised } \\
\text { subgroup) }\end{array}$ & $\begin{array}{l}\text { Bromate } \\
\text { (ozonation } \\
\text { DBP) }\end{array}$ & $\begin{array}{l}\text { Renal cell } \\
\text { cancer and } \\
\text { infections with } \\
\text { Cryptosporidiu } \\
\text { m Parvum }\end{array}$ & $\begin{array}{l}\text { Annual } \\
\text { averages of } \\
\text { raw water } \\
\text { data used - } \\
\text { can be } \\
\text { considered } \\
\text { as valid for } \\
\text { a typical } \\
\text { plant } \\
\text { operating } \\
\text { over one } \\
\text { year at the } \\
\text { time of } \\
\text { study } \\
\text { publication }\end{array}$ & $\begin{array}{l}\text { Comparison of } \\
\text { the risks of } \\
\text { disinfection } \\
\text { (chemical) } \\
\text { with its } \\
\text { benefits } \\
\text { (microbial) by } \\
\text { way of explicit } \\
\text { scenarios } \\
\text { relating to use } \\
\text { of ozonation } \\
\text { and an } \\
\text { immune- } \\
\text { compromised } \\
\text { population } \\
\text { subgroup }\end{array}$ & $\begin{array}{l}\text { Ingestion of } \\
\text { DW }\end{array}$ & \begin{tabular}{|l} 
Predictive \\
models used to \\
generate \\
estimates of \\
bromate and \\
microbial \\
contamination \\
at DWTP \\
Daily ingested \\
dose derived \\
from \\
concentration \\
multiplied by \\
typical daily \\
ingestion
\end{tabular} & $\begin{array}{l}\text { Both maximum } \\
\text { likelihood } \\
\text { estimate (MLE) } \\
\text { and } \\
\text { probabilistic } \\
\text { estimate of } \\
\text { dose-response } \\
\text { for renal cell } \\
\text { cancer based on } \\
\text { animal data } \\
\text { fitted with } \\
\text { linearised } \\
\text { multistage } \\
\text { model }\end{array}$ & $\begin{array}{l}\text { MLE of lifetime } \\
\text { excess risk } \\
\text { derived from the } \\
\text { animal model } \\
\text { used to predict } \\
\text { renal cell cancer } \\
\text { incidence at given } \\
\text { level of exposure } \\
\text { DALYs then } \\
\text { calculated as per } \\
\text { standard methods } \\
\text { using life } \\
\text { expectancies, } \\
\text { duration of } \\
\text { disease states and } \\
\text { severity } \\
\text { weightings }\end{array}$ & $\begin{array}{l}\text { MCS to account } \\
\text { for variation in } \\
\text { exposure and } \\
\text { response } \\
\text { parameters for } \\
\text { renal cell cancer } \\
\text { Additional animal } \\
\text { data fitted in } \\
\text { model to explore } \\
\text { heterogeneities } \\
\text { Univariate } \\
\text { sensitivity } \\
\text { analysis used to } \\
\text { investigate change } \\
\text { in model input } \\
\text { variables }\end{array}$ & $\begin{array}{l}\text { DALYs per } \\
\text { million } \\
\text { person years }\end{array}$ \\
\hline
\end{tabular}


Table 1 (continued)

\begin{tabular}{|c|c|c|c|c|c|c|c|c|c|c|c|}
\hline \multirow[b]{2}{*}{ Reference } & \multicolumn{5}{|c|}{ Issue framing } & \multicolumn{2}{|c|}{ Exposure assessment } & \multirow[b]{2}{*}{$\begin{array}{c}\text { Exposure- } \\
/ \text { dose-response }\end{array}$} & \multirow[b]{2}{*}{$\begin{array}{c}\text { Risk } \\
\text { characterisation }\end{array}$} & \multirow[b]{2}{*}{$\begin{array}{l}\text { Analysis of } \\
\text { uncertainty }\end{array}$} & \multirow[b]{2}{*}{$\begin{array}{l}\text { Outcome } \\
\text { metric }\end{array}$} \\
\hline & $\begin{array}{c}\text { Target } \\
\text { population }\end{array}$ & $\begin{array}{l}\text { Hazard/ } \\
\text { agent }\end{array}$ & Health outcome & $\begin{array}{l}\text { Temporal } \\
\text { framing }\end{array}$ & $\begin{array}{l}\text { Scenario(s) } \\
\text { considered }\end{array}$ & $\begin{array}{l}\text { Exposure } \\
\text { route }\end{array}$ & $\begin{array}{c}\text { Exposure } \\
\text { assessment } \\
\text { method }\end{array}$ & & & & \\
\hline (Hsu et al. 2001) & $\begin{array}{l}\text { Three areas } \\
\text { in different } \\
\text { regions of } \\
\text { Taiwan } \\
\text { supplied by } \\
3 \text { DWTPs }\end{array}$ & THM4 & $\begin{array}{l}\text { Cancer } \\
\text { (unspecified) }\end{array}$ & $\begin{array}{l}\text { Water } \\
\text { samples } \\
\text { taken } \\
\text { regularly } \\
\text { over period } \\
\text { 1994-1997 } \\
\text { Maximum } \\
\text { of } 30 \text { years } \\
\text { of exposure } \\
\text { assumed, } \\
\text { but lifetime } \\
\text { cancer risk } \\
\text { calculated }\end{array}$ & $\begin{array}{l}\text { Implicit } \\
\text { baseline of } \\
\text { zero exposure } \\
\text { to THMs }\end{array}$ & $\begin{array}{l}\text { Ingestion of } \\
\text { DW }\end{array}$ & $\begin{array}{l}\text { Geometric } \\
\text { means of THM } \\
\text { concentrations } \\
\text { (monitoring } \\
\text { data at } \\
\text { DWTPs) } \\
\\
\text { Age-adjusted } \\
\text { exposure } \\
\text { calculated from } \\
\text { generic } \\
\text { assumptions on } \\
\text { body weight, } \\
\text { consumption } \\
\text { rates etc. }\end{array}$ & $\begin{array}{l}\text { Published upper } \\
\text { bound } \mathrm{PF}_{\text {ingestion }}\end{array}$ & $\begin{array}{l}\text { Exposure } \\
\text { multiplied by } \\
\text { published PFs } \\
\\
\text { Rapid appraisal } \\
\text { and prioritisation } \\
\text { method applied } \\
\text { after (R. L. Smith } \\
\text { 1996) }\end{array}$ & N/a & $\begin{array}{l}\text { LECR due to } \\
\text { individual } \\
\text { and total } \\
\text { THMs }\end{array}$ \\
\hline $\begin{array}{l}\text { (Sadiq et al. } \\
\text { 2002) }\end{array}$ & $\begin{array}{l}\text { Two } \\
\text { neighbouring } \\
\text { towns in } \\
\text { Newfoundland, } \\
\text { Canada }\end{array}$ & $\mathrm{TCM}$ & $\begin{array}{l}\text { Cancer } \\
\text { (unspecified) }\end{array}$ & $\begin{array}{l}\text { Two } \\
\text { periods in } \\
1998 \\
\text { (summer } \\
\text { and winter); } \\
\text { exposure } \\
\text { estimates } \\
\text { extrapolated } \\
\text { over } \\
\text { lifetime }\end{array}$ & $\begin{array}{l}\text { DWTP with } \\
\text { and without } \\
\text { GAC }\end{array}$ & $\begin{array}{l}\text { Ingestion } \\
\text { (simulated } \\
\text { with PDFs of } \\
\text { generic } \\
\text { population } \\
\text { physical } \\
\text { parameters) }\end{array}$ & $\begin{array}{l}\text { Sampling } \\
\text { survey } \\
\text { throughout a } \\
\text { DW } \\
\text { distribution } \\
\text { network } \\
\\
\text { Bootstrapping } \\
\text { used to infer } \\
\text { robust non- } \\
\text { parametric } \\
\text { PDFs from } \\
\text { these data }\end{array}$ & $\begin{array}{l}\text { Published upper } \\
\text { bound } \mathrm{PF}_{\text {ingestion }}\end{array}$ & $\begin{array}{l}\text { Chronic daily } \\
\text { ingestion } \\
\text { multiplied by PF }\end{array}$ & $\begin{array}{l}\begin{array}{l}\text { Bootstrapping } \\
\text { (exposure } \\
\text { assessment) }\end{array} \\
\text { MCS (exposure } \\
\text { assessment and } \\
\text { risk } \\
\text { characterisation) }\end{array}$ & $\begin{array}{l}\text { LECR due to } \\
\text { ingested } \\
\text { TCM }\end{array}$ \\
\hline $\begin{array}{l}\text { (Williams et al. } \\
\text { 2002) }\end{array}$ & $\begin{array}{l}\text { California, } \\
\text { USA }\end{array}$ & $\begin{array}{l}\text { TCM (as one } \\
\text { of } 6 \text { VOCs) in } \\
\text { DW and raw } \\
\text { water (from } \\
\text { groundwater } \\
\text { sources) }\end{array}$ & $\begin{array}{l}\text { Cancer } \\
\text { (unspecified) }\end{array}$ & $1995-2001$ & $\begin{array}{l}\text { Comparison of } \\
\text { cancer risks } \\
\text { adjusted for } \\
\text { detection } \\
\text { frequency of } \\
\text { the VOCs with } \\
\text { unadjusted }\end{array}$ & $\begin{array}{l}\text { Multipathway } \\
\text { (ingestion } \\
\text { of DW, } \\
\text { dermal } \\
\text { contact and } \\
\text { inhalation of } \\
\text { DBPs via } \\
\text { showering) }\end{array}$ & $\begin{array}{l}\text { State wide } \\
\text { sampling } \\
\text { survey of } \\
\sim 2500 \mathrm{DW} \\
\text { systems (mean } \\
\text { detected } \\
\text { concentrations) }\end{array}$ & $\begin{array}{l}\text { Published upper } \\
\text { bound PFs }\end{array}$ & $\begin{array}{l}\text { Potential LCR } \\
\text { calculated as } \\
\text { LADD multiplied } \\
\text { by PF }\end{array}$ & N/a & $\begin{array}{l}\text { Relative } \\
\text { lifetime } \\
\text { cancer risk }\end{array}$ \\
\hline (Fehr et al. 2003) & $\begin{array}{l}\text { Population } \\
\text { of North } \\
\text { Rhine- } \\
\text { Westphalia, } \\
\text { Germany }\end{array}$ & $\begin{array}{l}\text { TCM (as one } \\
\text { of } 8 \\
\text { carcinogenic } \\
\text { chemical } \\
\text { contaminants } \\
\text { in water) }\end{array}$ & $\begin{array}{l}\text { Cancer } \\
\text { (unspecified) }\end{array}$ & $\begin{array}{l}\text { Not } \\
\text { specified, } \\
\text { but } \\
\text { exposure in } \\
\text { baseline } \\
\text { scenario } \\
\text { based on } \\
\text { 'current' } \\
\text { monitoring } \\
\text { data; } \\
\text { exposure } \\
\text { estimates } \\
\text { extrapolated } \\
\text { over } \\
\text { lifetime }\end{array}$ & $\begin{array}{l}\text { Comparison of } \\
2 \text { water } \\
\text { privatisation } \\
\text { policies: } \\
\text { business-as- } \\
\text { usual (public } \\
\text { water supply) } \\
\text { vs. privatised } \\
\text { water supply }\end{array}$ & $\begin{array}{l}\text { Ingestion of } \\
\text { DW (CDI } \\
\text { calculated } \\
\text { using PDFs } \\
\text { for body } \\
\text { weight and } \\
\text { water intake) }\end{array}$ & $\begin{array}{l}\text { Baseline } \\
\text { scenario } \\
\text { exposure based } \\
\text { on current } \\
\text { levels of } \\
\text { contaminants. } \\
\text { Privatisation } \\
\text { scenario } \\
\text { contaminant } \\
\text { levels based on } \\
\text { percentage } \\
\text { exhaustion of } \\
\text { regulatory } \\
\text { limits }\end{array}$ & $\begin{array}{l}\text { Published upper } \\
\text { bound } \mathrm{PF}_{\text {ingestion, }} \\
\text { specified as a) a } \\
\text { point estimate } \\
\text { and b) a PDF } \\
\\
\text { Values of } \\
\text { PF } \\
\text { provestion not }\end{array}$ & $\begin{array}{l}\text { Deterministic and } \\
\text { probabilistic } \\
\text { estimation of risk } \\
\text { by multiplying } \\
\text { PFs by exposure } \\
\text { distributions } \\
\text { Unit risks for all } \\
\text { carcinogens were } \\
\text { summed and } \\
\text { multiplied by the } \\
\text { entire population }\end{array}$ & $\begin{array}{l}\text { MCS (exposure } \\
\text { assessment) } \\
\text { Sensitivity } \\
\text { analysis based on } \\
\text { deterministic and } \\
\text { probabilistic } \\
\text { methods in } \\
\text { assessment of } \\
\text { exposure and } \\
\text { definitions of PFs }\end{array}$ & $\begin{array}{l}\text { LECC due to } \\
\text { increase in } \\
\text { carcinogens } \\
\text { (including } \\
\text { ingestion of } \\
\text { TCM ) }\end{array}$ \\
\hline $\begin{array}{l}\text { (Sofuoglu et al. } \\
2003 \text { ) }\end{array}$ & $\begin{array}{l}\text { Two } \\
\text { populations } \\
\text { in Arizona, } \\
\text { USA }\end{array}$ & $\begin{array}{l}\text { TCM (as one } \\
\text { of a suite of } \\
\text { contaminants, } \\
\text { including } \\
\text { metals, } \\
\text { volatile } \\
\text { organic } \\
\text { compounds } \\
\text { (VOCs) and } \\
\text { pesticides }\end{array}$ & $\begin{array}{l}\text { Cancer } \\
\text { (unspecified) }\end{array}$ & $\begin{array}{l}\text { NHEXAS } \\
\text { data from } \\
1998\end{array}$ & $\begin{array}{l}\text { Implicit } \\
\text { baseline of } \\
\text { zero exposure } \\
\text { to THMs } \\
\text { Comparison } \\
\text { between } 2 \\
\text { areas with } \\
\text { different } \\
\text { hypothesized } \\
\text { difference in } \\
\text { environmental } \\
\text { quality }\end{array}$ & $\begin{array}{l}\text { Ingestion of } \\
\text { DW }\end{array}$ & $\begin{array}{l}\text { NHEXAS } \\
\text { Arizona } \\
\text { sampling study } \\
\text { (DW from } \\
\text { households) } \\
\text { Annual daily } \\
\text { ingestion } \\
\text { estimated using } \\
\text { probabilistic } \\
\text { simulation }\end{array}$ & $\begin{array}{l}\text { Published upper } \\
\text { bound } \mathrm{PF}_{\text {ingestion }} \\
\text { Values not } \\
\text { provided }\end{array}$ & $\begin{array}{l}\text { Product of PF and } \\
\text { annual daily } \\
\text { exposure }\end{array}$ & $\begin{array}{l}\text { Bootstrapping and } \\
\text { MCS (exposure } \\
\text { assessment) }\end{array}$ & $\begin{array}{l}\text { LECR due to } \\
\text { ingestion of } \\
\text { TCM }\end{array}$ \\
\hline (Lee et al. 2004) & $\begin{array}{l}19 \text { districts } \\
\text { of Hong } \\
\text { Kong }\end{array}$ & THM4 & $\begin{array}{l}\text { Cancer } \\
\text { (unspecified) } \\
\text { and non-cancer } \\
\text { outcomes } \\
\text { (unspecified) }\end{array}$ & $\begin{array}{l}\text { Temporal } \\
\text { frame of } \\
\text { sampling } \\
\text { survey not } \\
\text { presented; } \\
\text { annual } \\
\text { excess } \\
\text { cases of } \\
\text { cancer }\end{array}$ & $\begin{array}{l}\text { Implicit } \\
\text { baseline of } \\
\text { zero exposure } \\
\text { to THMs }\end{array}$ & $\begin{array}{l}\text { Multipathway } \\
\text { (ingestion } \\
\text { of DW, } \\
\text { dermal } \\
\text { contact and } \\
\text { inhalation of } \\
\text { DBPs via } \\
\text { showering) }\end{array}$ & $\begin{array}{l}\text { Measured } \\
\text { THM4 across } \\
\text { DW } \\
\text { distribution } \\
\text { network } \\
\\
\text { CDIs calculated } \\
\text { using generic } \\
\text { US population } \\
\text { parameters }\end{array}$ & $\begin{array}{l}\text { Published upper } \\
\text { bound PFs } \\
\text { RfDs for oral } \\
\text { and dermal } \\
\text { routes }\end{array}$ & $\begin{array}{l}\text { Sum of exposure- } \\
\text { route specific } \\
\text { LECR calculated } \\
\text { as chronic daily } \\
\text { exposure } \\
\text { multiplied by PFs } \\
\text { for each THM } \\
\text { after (Gratt 1996). } \\
\text { HI calculated } \\
\text { assuming risk } \\
\text { additivity }\end{array}$ & $\mathrm{N} / \mathrm{a}$ & $\begin{array}{l}\text { Annual } \\
\text { excess cases } \\
\text { of cancer and } \\
\text { HI due to } \\
\text { multipathway } \\
\text { exposure to } \\
\text { THM4 }\end{array}$ \\
\hline $\begin{array}{l}\text { (Tokmak et al. } \\
\text { 2004) }\end{array}$ & $\begin{array}{l}\text { Ankara, } \\
\text { Turkey }\end{array}$ & THM4 & $\begin{array}{l}\text { Cancer } \\
\text { (unspecified) }\end{array}$ & $\begin{array}{l}\text { Temporal } \\
\text { frame of } \\
\text { sampling } \\
\text { survey not } \\
\text { presented; } \\
\text { annual } \\
\text { excess } \\
\text { cases of } \\
\text { cancer }\end{array}$ & $\begin{array}{l}\text { Implicit } \\
\text { baseline of } \\
\text { zero exposure } \\
\text { to THMs }\end{array}$ & $\begin{array}{l}\text { Multipathway } \\
\text { (ingestion } \\
\text { of DW, } \\
\text { dermal } \\
\text { contact and } \\
\text { inhalation of } \\
\text { DBPs via } \\
\text { showering) }\end{array}$ & $\begin{array}{l}\begin{array}{l}\text { Samples of } \\
\text { consumer tap } \\
\text { water in } 22 \\
\text { districts }\end{array} \\
\text { Estimation of } \\
\text { exposure after } \\
\text { Lee et al. } \\
(2004)\end{array}$ & $\begin{array}{l}\text { Upper bound } \\
\text { PFs and RfDs } \\
\text { from Lee et al. } \\
(2004)\end{array}$ & $\begin{array}{l}\text { Method after Lee } \\
\text { et al. (2004) }\end{array}$ & $\mathrm{N} / \mathrm{a}$ & $\begin{array}{l}\text { Annual } \\
\text { excess cases } \\
\text { of cancer and } \\
\text { HI due to } \\
\text { multipathway } \\
\text { exposure to } \\
\text { THM4 }\end{array}$ \\
\hline
\end{tabular}


Table 1 (continued)

\begin{tabular}{|c|c|c|c|c|c|c|c|c|c|c|c|}
\hline \multirow[b]{2}{*}{ Reference } & \multicolumn{5}{|c|}{ Issue framing } & \multicolumn{2}{|c|}{ Exposure assessment } & \multirow[b]{2}{*}{$\begin{array}{c}\text { Exposure- } \\
\text { /dose-response }\end{array}$} & \multirow[b]{2}{*}{$\begin{array}{c}\text { Risk } \\
\text { characterisation }\end{array}$} & \multirow[b]{2}{*}{$\begin{array}{l}\text { Analysis of } \\
\text { uncertainty }\end{array}$} & \multirow[b]{2}{*}{$\begin{array}{l}\text { Outcome } \\
\text { metric }\end{array}$} \\
\hline & $\begin{array}{c}\text { Target } \\
\text { population }\end{array}$ & $\begin{array}{c}\text { Hazard/ } \\
\text { agent }\end{array}$ & Health outcome & $\begin{array}{l}\text { Temporal } \\
\text { framing }\end{array}$ & $\begin{array}{l}\text { Scenario(s) } \\
\text { considered }\end{array}$ & $\begin{array}{l}\text { Exposure } \\
\text { route }\end{array}$ & $\begin{array}{c}\text { Exposure } \\
\text { assessment } \\
\text { method }\end{array}$ & & & & \\
\hline (USEPA 2005) & $\begin{array}{l}\text { Population } \\
\text { of US } \\
\text { potentially } \\
\text { exposed to } \\
\text { DBPs from } \\
\text { drinking } \\
\text { water ( 263 } \\
\text { million) } \\
\text { Subgroup } \\
\text { analyses } \\
\text { carried out } \\
\text { for women } \\
\text { of child- } \\
\text { bearing age } \\
\text { and } \\
\text { developing } \\
\text { foetuses }\end{array}$ & $\begin{array}{l}\text { TTHM and } \\
\text { HAA5 }\end{array}$ & $\begin{array}{l}\text { Bladder cancer, } \\
\text { colon cancer, } \\
\text { adverse birth } \\
\text { outcomes } \\
\text { (specifically } \\
\text { foetal losses) }\end{array}$ & \begin{tabular}{|l|} 
Temporal \\
variability \\
accounted \\
for in \\
exposure \\
models \\
\\
"Cessation \\
lag" \\
function \\
used to \\
project \\
bladder \\
cancer \\
cases \\
avoided \\
each \\
year after \\
implementation \\
as a result of \\
the Stage 2 \\
DBPR over \\
a 100-year \\
period
\end{tabular} & $\begin{array}{l}\text { Four } \\
\text { alternatives } \\
\text { relating to } \\
\text { regulatory } \\
\text { requirements } \\
\text { on drinking } \\
\text { water } \\
\text { providers } \\
\text { (Stage 2 } \\
\text { DBPR) }\end{array}$ & 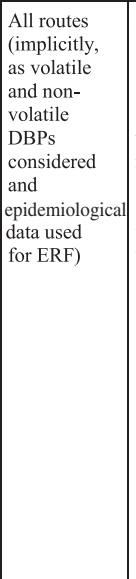 & $\begin{array}{l}\text { Modelling of } \\
\text { drinking water } \\
\text { concentrations } \\
\text { of DBPs, } \\
\text { incorporating } \\
\text { uncertainties } \\
\text { relating to } \\
\text { compliance, } \\
\text { spatial and } \\
\text { temporal } \\
\text { variability }\end{array}$ & $\begin{array}{l}\text { Epidemiologica } \\
1 \text { data from five } \\
\text { studies } \\
\\
\text { (Toxicological } \\
\text { data used for } \\
\text { comparison } \\
\text { only) }\end{array}$ & $\begin{array}{l}\text { PAR, PAC (cases } \\
\text { of cancer etc. } \\
\text { avoided), dollars } \\
\text { (LCR from } \\
\text { toxicological data } \\
\text { for comparison } \\
\text { only) }\end{array}$ & $\begin{array}{l}\text { Extensive } \\
\text { uncertainty } \\
\text { analysis: } \\
\text { quantitative } \\
\text { (MCS, sensitivity } \\
\text { analysis, } \\
\text { alternative } \\
\text { models, } \\
\text { populations, data } \\
\text { etc.) and } \\
\text { qualitative (lists of } \\
\text { uncertainties and } \\
\text { their potential } \\
\text { effects) }\end{array}$ & $\begin{array}{l}\text { Monetised } \\
\text { costs and } \\
\text { benefits }\end{array}$ \\
\hline $\begin{array}{l}\text { (Kavcar et al. } \\
2006)\end{array}$ & $\begin{array}{l}\text { Izmir, } \\
\text { Turkey }\end{array}$ & $\begin{array}{l}\text { THM4 (as one } \\
\text { group of } \\
\text { several VOCs) } \\
\text { in tap water } \\
\text { and bottled } \\
\text { water }\end{array}$ & $\begin{array}{l}\text { Cancer } \\
\text { (unspecified) }\end{array}$ & $\begin{array}{l}\text { Not } \\
\text { presented; } \\
\text { lifetime } \\
\text { cancer risk }\end{array}$ & $\begin{array}{l}\text { Implicit } \\
\text { baseline of } \\
\text { zero exposure } \\
\text { to THMs } \\
\text { Comparison of } \\
\text { tap water and } \\
\text { bottled water }\end{array}$ & $\begin{array}{l}\text { Ingestion of } \\
\text { DW }\end{array}$ & $\begin{array}{l}\text { Sampling } \\
\text { survey of } 100 \\
\text { households }\end{array}$ & $\begin{array}{l}\text { Published upper } \\
\text { bound } \mathrm{PF}_{\text {ingestion }} \\
\text { RfD used } \\
\text { instead of PF } \\
\text { for TCM }\end{array}$ & $\begin{array}{l}\text { Chronic daily } \\
\text { ingested dose } \\
\text { multiplied by PF } \\
\text { RfD used to } \\
\text { calculate HQ for } \\
\text { TCM as } \\
\text { secondary } \\
\text { carcinogen }\end{array}$ & $\begin{array}{l}\text { MCS (exposure } \\
\text { assessment) }\end{array}$ & $\begin{array}{l}\text { LECR due to } \\
\text { ingestion of } \\
\text { THM4 }\end{array}$ \\
\hline $\begin{array}{l}\text { (Nazir \& Khan } \\
\text { 2006) }\end{array}$ & $\begin{array}{l}\text { Three } \\
\text { communities } \\
\text { in } \\
\text { Newfoundland, } \\
\text { Canada }\end{array}$ & $\begin{array}{l}\text { TCM (as a } \\
\text { "representative } \\
\text { chemical" } \\
\text { for the THM } \\
\text { mixture) }\end{array}$ & $\begin{array}{l}\text { Cancer } \\
\text { (unspecified) }\end{array}$ & $\begin{array}{l}\text { Summer } \\
\text { and winter } \\
\text { in one year; } \\
\text { averaged } \\
\text { over } \\
\text { lifetime }\end{array}$ & $\begin{array}{l}\text { Comparison of } \\
\text { shower design } \\
\text { parameters and } \\
\text { shower usage }\end{array}$ & \begin{tabular}{|l} 
Multipathway \\
(ingestion \\
of DW, \\
dermal \\
contact and \\
inhalation of \\
DBPs via \\
showering)
\end{tabular} & $\begin{array}{l}\text { Process-based } \\
\text { modelling of } \\
\text { inhalation and } \\
\text { dermal contact } \\
\text { during } \\
\text { showering after } \\
\text { Jo et al. (1990) }\end{array}$ & $\begin{array}{l}\text { Published upper } \\
\text { bound } \mathrm{PF}_{\text {inhalation }} \\
\text { through } \\
\text { inhalation } \\
\text { Published oral } \\
\text { RfD used } \\
\text { instead of PF } \\
\text { for ingestion } \\
\text { and dermal }\end{array}$ & $\begin{array}{l}\text { Modelled } \\
\text { probabilistic } \\
\text { exposure } \\
\text { estimates } \\
\text { multiplied by PF } \\
\text { (or RfD) } \\
\text { RfD used to } \\
\text { calculate HQ for } \\
\text { TCM as } \\
\text { secondary } \\
\text { carcinogen }\end{array}$ & $\begin{array}{l}\text { MCS (exposure } \\
\text { assessment) } \\
\text { Two alternative } \\
\text { models of shower } \\
\text { air THM } \\
\text { concentrations }\end{array}$ & $\begin{array}{l}\text { LECR due to } \\
\text { multi- } \\
\text { pathway } \\
\text { exposure to } \\
\text { TCM }\end{array}$ \\
\hline (Uyak 2006) & $\begin{array}{l}\text { Istanbul, } \\
\text { Turkey }\end{array}$ & THM4 & $\begin{array}{l}\text { Cancer } \\
\text { (unspecified) } \\
\text { and non-cancer } \\
\text { outcomes } \\
\text { (unspecific) }\end{array}$ & $\begin{array}{l}\text { 24-week } \\
\text { sampling } \\
\text { survey } \\
\text { (summer, } \\
2004) ; \\
\text { averaged } \\
\text { over } \\
\text { lifetime }\end{array}$ & $\begin{array}{l}\text { Implicit } \\
\text { baseline of } \\
\text { zero exposure } \\
\text { to THMs }\end{array}$ & \begin{tabular}{|l|} 
Multipathway \\
(ingestion \\
of DW, \\
dermal \\
contact and \\
inhalation of \\
DBPs via \\
showering)
\end{tabular} & $\begin{array}{l}\text { Sampling of } \\
\text { household tap } \\
\text { water } \\
\\
\text { Exposure } \\
\text { assessed after } \\
\text { Lee et al. } \\
\text { (2004) }\end{array}$ & $\begin{array}{l}\text { Published upper } \\
\text { bound PFs } \\
\text { (including } \\
\text { rescinded data } \\
\text { for TCM) and } \\
\text { RfDs }\end{array}$ & $\begin{array}{l}\text { Method after (S. } \\
\text { C. Lee et al. } \\
\text { 2004) } \\
\text { Excess cases } \\
\text { calculated as } \\
\text { lifetime cancer } \\
\text { risk multiplied by } \\
\text { Istanbul } \\
\text { population }\end{array}$ & $\mathrm{N} / \mathrm{a}$ & $\begin{array}{l}\text { Annual } \\
\text { excess cases } \\
\text { of cancer and } \\
\text { HI due to } \\
\text { multipathway } \\
\text { exposure to } \\
\text { THM4 }\end{array}$ \\
\hline $\begin{array}{l}\text { (Aslan \& } \\
\text { Turkman 2007) }\end{array}$ & $\begin{array}{l}\text { Izmir, } \\
\text { Turkey }\end{array}$ & THM4 & $\begin{array}{l}\text { Cancer } \\
\text { (unspecified) } \\
\text { and non-cancer } \\
\text { outcomes } \\
\text { (unspecified) }\end{array}$ & $\begin{array}{l}\text { One-year } \\
\text { survey; } \\
\text { averaged } \\
\text { over } \\
\text { lifetime }\end{array}$ & $\begin{array}{l}\text { Implicit } \\
\text { baseline of } \\
\text { zero exposure } \\
\text { to THMs }\end{array}$ & $\begin{array}{l}\text { Multipathway } \\
\text { (ingestion } \\
\text { of DW, } \\
\text { dermal } \\
\text { contact and } \\
\text { inhalation of } \\
\text { DBPs via } \\
\text { showering) }\end{array}$ & $\begin{array}{l}\text { Water samples } \\
\text { at DWTP } \\
\text { Exposure } \\
\text { assessed after } \\
\text { (S. C. Lee et al. } \\
\text { 2004) }\end{array}$ & $\begin{array}{l}\text { Published upper } \\
\text { bound PFs } \\
\text { (including } \\
\text { rescinded data } \\
\text { for TCM) }\end{array}$ & $\begin{array}{l}\text { Method after (S. } \\
\text { C. Lee et al. } \\
\text { 2004) }\end{array}$ & N/a & $\begin{array}{l}\text { LECR and HI } \\
\text { due to } \\
\text { multipathway } \\
\text { exposure to } \\
\text { TCM }\end{array}$ \\
\hline $\begin{array}{l}\text { (Navarro et al. } \\
2007 \text { ) }\end{array}$ & $\begin{array}{l}\text { Four } \\
\text { communities } \\
\text { in central } \\
\text { Mexico }\end{array}$ & THM4 & $\begin{array}{l}\text { Cancer } \\
\text { (unspecified) } \\
\text { and non-cancer } \\
\text { outcomes } \\
\text { (unspecified) }\end{array}$ & $\begin{array}{l}\text { 2-week } \\
\text { sampling } \\
\text { program in } \\
\text { May } 2005\end{array}$ & $\begin{array}{l}\text { Implicit } \\
\text { baseline of } \\
\text { zero exposure } \\
\text { to THMs }\end{array}$ & $\begin{array}{l}\text { Ingestion of } \\
\text { DW }\end{array}$ & $\begin{array}{l}\text { Sampling of } \\
\text { household tap } \\
\text { water }\end{array}$ & $\begin{array}{l}\text { Published upper } \\
\text { bound PFs for } \\
\text { brominated } \\
\text { THMs. RfD } \\
\text { used for TCM }\end{array}$ & $\begin{array}{l}\begin{array}{l}\text { Chronic daily } \\
\text { ingestion } \\
\text { multiplied by PFs } \\
\text { (bromide THMs) }\end{array} \\
\text { RfD used to } \\
\text { calculate HQ for } \\
\text { TCM as } \\
\text { secondary } \\
\text { carcinogen }\end{array}$ & MCS (exposure) & $\begin{array}{l}\text { LECR due to } \\
\text { ingestion of } \\
\text { THM4 }\end{array}$ \\
\hline $\begin{array}{l}\text { (Semerjian \& } \\
\text { Dennis 2007) }\end{array}$ & Lebanon & THM4 & $\begin{array}{l}\text { Cancer } \\
\text { (unspecified) } \\
\text { and non-cancer } \\
\text { outcomes } \\
\text { (unspecified) }\end{array}$ & $\begin{array}{l}\text { One year } \\
\text { sampling } \\
\text { survey } \\
\text { (spring, } \\
\text { summer, } \\
\text { winter) }\end{array}$ & $\begin{array}{l}\text { Implicit } \\
\text { baseline of } \\
\text { zero exposure } \\
\text { to THMs }\end{array}$ & 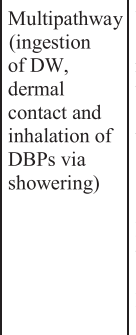 & $\begin{array}{l}\text { Water samples } \\
\text { "collected } \\
\text { randomly from } \\
\text { various water } \\
\text { sources and } \\
\text { water } \\
\text { distribution } \\
\text { systems" } \\
\text { LADDs were } \\
\text { then calculated, } \\
\text { according to } \\
\text { Lee et al. } \\
\text { (2004) }\end{array}$ & $\begin{array}{l}\text { Published upper } \\
\text { bound PFs } \\
\text { (including } \\
\text { rescinded data } \\
\text { for TCM) }\end{array}$ & $\begin{array}{l}\text { Two approaches } \\
\text { used: (1) WHO } \\
\text { index for additive } \\
\text { toxicity; and (2) } \\
\text { USEPA-approved } \\
\text { Risk Assistant } \\
\text { model }\end{array}$ & $\begin{array}{l}\text { Sensitivity } \\
\text { analysis of } 2 \\
\text { methods of risk } \\
\text { characterisation }\end{array}$ & $\begin{array}{l}\text { LECR and HI } \\
\text { due to } \\
\text { multipathway } \\
\text { exposure to } \\
\text { THM4 }\end{array}$ \\
\hline
\end{tabular}


Table 1 (continued)

\begin{tabular}{|c|c|c|c|c|c|c|c|c|c|c|c|}
\hline \multirow[b]{2}{*}{ Reference } & \multicolumn{5}{|c|}{ Issue framing } & \multicolumn{2}{|c|}{ Exposure assessment } & \multirow[b]{2}{*}{$\begin{array}{c}\text { Exposure- } \\
\text { /dose-response }\end{array}$} & \multirow[b]{2}{*}{$\begin{array}{c}\text { Risk } \\
\text { characterisation }\end{array}$} & \multirow[b]{2}{*}{$\begin{array}{l}\text { Analysis of } \\
\text { uncertainty }\end{array}$} & \multirow[b]{2}{*}{$\begin{array}{c}\text { Outcome } \\
\text { metric }\end{array}$} \\
\hline & $\begin{array}{c}\text { Target } \\
\text { population }\end{array}$ & $\begin{array}{l}\text { Hazard/ } \\
\text { agent }\end{array}$ & Health outcome & $\begin{array}{l}\text { Temporal } \\
\text { framing }\end{array}$ & $\begin{array}{l}\text { Scenario(s) } \\
\text { considered }\end{array}$ & $\begin{array}{l}\text { Exposure } \\
\text { route }\end{array}$ & $\begin{array}{c}\text { Exposure } \\
\text { assessment } \\
\text { method }\end{array}$ & & & & \\
\hline $\begin{array}{l}\text { (G. S. Wang et } \\
\text { al. 2007) }\end{array}$ & $\begin{array}{l}\text { Three } \\
\text { metropolitan } \\
\text { districts of } \\
\text { Taiwan, and } \\
\text { one island } \\
\text { county } \\
\text { (Kinmen) }\end{array}$ & THM4 & $\begin{array}{l}\text { Cancer } \\
\text { (unspecified) }\end{array}$ & $\begin{array}{l}\text { One year of } \\
\text { sampling } \\
\text { between } \\
\text { July } 2002 \\
\text { and May } \\
2003\end{array}$ & $\begin{array}{l}\text { Implicit } \\
\text { baseline of } \\
\text { zero exposure } \\
\text { to THMs }\end{array}$ & $\begin{array}{l}\text { Multipathway } \\
\text { (ingestion } \\
\text { of DW, } \\
\text { dermal } \\
\text { contact and } \\
\text { inhalation of } \\
\text { DBPs via } \\
\text { showering) }\end{array}$ & 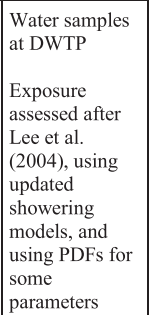 & $\begin{array}{l}\text { Published upper } \\
\text { bound PFs } \\
\text { (including } \\
\text { rescinded data } \\
\text { for TCM) }\end{array}$ & $\begin{array}{l}\text { Method after (S. } \\
\text { C. Lee et al. } \\
\text { 2004) }\end{array}$ & $\begin{array}{l}\text { MCS (exposure) } \\
\text { Sensitivity } \\
\text { analysis on effect } \\
\text { of using PFs in } \\
\text { exposure } \\
\text { pathways for } \\
\text { which they were } \\
\text { not specifically } \\
\text { intended }\end{array}$ & $\begin{array}{l}\text { LECR due to } \\
\text { multipathway } \\
\text { exposure to } \\
\text { THM4 }\end{array}$ \\
\hline $\begin{array}{l}\text { (W. Wang et al. } \\
2007 \text { ) }\end{array}$ & $\begin{array}{l}\text { Fifteen } \\
\text { waterworks } \\
\text { in Beijing, } \\
\text { China and } \\
\text { three } \\
\text { DWTPs in } \\
\text { Canada }\end{array}$ & $\begin{array}{l}\text { THM4 and } \\
\text { haloacetic } \\
\text { acids }\end{array}$ & $\begin{array}{l}\text { Cancer } \\
\text { (unspecified) } \\
\text { and non-cancer } \\
\text { outcomes } \\
\text { (unspecified) }\end{array}$ & $\begin{array}{l}\begin{array}{l}\text { China: } \\
\text { one-off } \\
\text { sampling } \\
\text { survey }\end{array} \\
\text { Canada: } \\
\text { averages of } \\
\text { monthly } \\
\text { measurements } \\
\text { over 1 year }\end{array}$ & $\begin{array}{l}\text { Implicit } \\
\text { baseline of } \\
\text { zero exposure } \\
\text { to THMs } \\
\text { Comparison of } \\
\text { risks in } 2 \\
\text { countries } \\
\text { Comparison of } \\
\text { risks under } \\
\text { three types of } \\
\text { treatment in } \\
\text { Canada }\end{array}$ & $\begin{array}{l}\text { Multipathway } \\
\text { (ingestion } \\
\text { of DW, } \\
\text { dermal } \\
\text { contact and } \\
\text { inhalation of } \\
\text { DBPs via } \\
\text { showering) }\end{array}$ & $\begin{array}{l}\text { Water samples } \\
\text { at DWTP } \\
\text { Exposure } \\
\text { assessed after } \\
\text { Lee et al. } \\
(2004)\end{array}$ & $\begin{array}{l}\text { Published upper } \\
\text { bound PFs } \\
\text { (including } \\
\text { rescinded data } \\
\text { for TCM) }\end{array}$ & $\begin{array}{l}\text { Method after (S. } \\
\text { C. Lee et al. } \\
\text { 2004) } \\
\text { Excess cases } \\
\text { calculated as } \\
\text { hazard index } \\
\text { multiplied by } \\
\text { appropriate } \\
\text { populations }\end{array}$ & $\begin{array}{l}\text { A list of sources } \\
\text { of uncertainty was } \\
\text { presented, in } \\
\text { particular } \\
\text { recognising that } \\
\text { estimates are } \\
\text { upper bound }\end{array}$ & $\begin{array}{l}\text { LECR and HI } \\
\text { due to } \\
\text { multipathway } \\
\text { exposure to } \\
\text { THM4 }\end{array}$ \\
\hline $\begin{array}{l}\text { (Baytak et al. } \\
\text { 2008) }\end{array}$ & $\begin{array}{l}\text { Izmir, } \\
\text { Turkey }\end{array}$ & THM4 & $\begin{array}{l}\text { Cancer } \\
\text { (unspecified) } \\
\text { and non-cancer } \\
\text { outcomes } \\
\text { (unspecified) }\end{array}$ & $\begin{array}{l}\text { Sampling } \\
\text { survey } \\
\text { carried out } \\
\text { between } \\
\text { July } 2006 \\
\text { and April } \\
2007\end{array}$ & $\begin{array}{l}\text { Implicit } \\
\text { baseline of } \\
\text { zero exposure } \\
\text { to THMs }\end{array}$ & $\begin{array}{l}\text { Ingestion of } \\
\text { DW }\end{array}$ & $\begin{array}{l}\text { Tap water } \\
\text { samples taken } \\
\text { across five } \\
\text { districts of } \\
\text { Izmir } \\
\\
\text { Exposure } \\
\text { assessed after } \\
\text { Lee et al. } \\
\text { (2004), with } \\
\text { body weight } \\
\text { and water } \\
\text { consumption } \\
\text { represented } \\
\text { probabilistically }\end{array}$ & $\begin{array}{l}\text { Published upper } \\
\text { bound PFs } \\
\begin{array}{l}\text { Oral RfD used } \\
\text { for TCM }\end{array}\end{array}$ & $\begin{array}{l}\text { Method after (S. } \\
\text { C. Lee et al. } \\
\text { 2004) } \\
\text { RfD used to } \\
\text { calculate HQ for } \\
\text { TCM as } \\
\text { secondary } \\
\text { carcinogen }\end{array}$ & MCS (exposure) & $\begin{array}{l}\text { Probabilistic } \\
\text { estimates of } \\
\text { LECR and HI } \\
\text { due to } \\
\text { multipathway } \\
\text { exposure to } \\
\text { THM4 }\end{array}$ \\
\hline $\begin{array}{l}\text { (Viana et al. } \\
\text { 2009) }\end{array}$ & $\begin{array}{l}\text { Ten } \\
\text { metropolitan } \\
\text { districts of } \\
\text { Fortaleza, } \\
\text { Brazil }\end{array}$ & THM4 & $\begin{array}{l}\text { Cancer } \\
\text { (unspecified) } \\
\text { and non-cancer } \\
\text { outcomes } \\
\text { (unspecified) }\end{array}$ & \begin{tabular}{l|} 
Sampling \\
survey \\
carried out \\
between \\
October and \\
December \\
2004
\end{tabular} & $\begin{array}{l}\text { Implicit } \\
\text { baseline of } \\
\text { zero exposure } \\
\text { to THMs }\end{array}$ & $\begin{array}{l}\text { Multipathway } \\
\text { (ingestion } \\
\text { of DW, } \\
\text { dermal } \\
\text { contact and } \\
\text { inhalation of } \\
\text { DBPs via } \\
\text { showering) }\end{array}$ & $\begin{array}{l}\begin{array}{l}\text { Sampling of } \\
\text { household tap } \\
\text { water }\end{array} \\
\text { Exposure } \\
\text { assessed after } \\
\text { Lee et al. } \\
(2004)\end{array}$ & $\begin{array}{l}\text { Published upper } \\
\text { bound PFs and } \\
\text { RfDs } \\
\text { No values } \\
\text { presented }\end{array}$ & $\begin{array}{l}\text { Method after (S. } \\
\text { C. Lee et al. } \\
\text { 2004) }\end{array}$ & $\mathrm{N} / \mathrm{a}$ & $\begin{array}{l}\text { LECR and HI } \\
\text { due to } \\
\text { multipathway } \\
\text { exposure to } \\
\text { THM4 }\end{array}$ \\
\hline $\begin{array}{l}\text { (Chowdhury \& } \\
\text { Champagne } \\
\text { 2009) }\end{array}$ & $\begin{array}{l}\text { Three major } \\
\text { cities in } \\
\text { Ontario, } \\
\text { Canada }\end{array}$ & THM4 & $\begin{array}{l}\text { Cancer } \\
\text { (unspecified) } \\
\text { and non-cancer } \\
\text { outcomes } \\
\text { (unspecified) }\end{array}$ & $\begin{array}{l}2000-2004 ; \\
\text { annual } \\
\text { cancer risk } \\
\text { estimated }\end{array}$ & $\begin{array}{l}\begin{array}{l}\text { Variable } \\
\text { shower stall } \\
\text { volumes and } \\
\text { durations }\end{array} \\
\text { Implicit } \\
\text { baseline of } \\
\text { zero exposure } \\
\text { to THMs }\end{array}$ & $\begin{array}{l}\text { Multipathway } \\
\text { (ingestion } \\
\text { of DW, } \\
\text { dermal } \\
\text { contact and } \\
\text { inhalation of } \\
\text { DBPs via } \\
\text { showering) }\end{array}$ & $\begin{array}{l}\text { Sampling of } \\
\text { household tap } \\
\text { water } \\
\text { Process-based } \\
\text { modelling of } \\
\text { inhalation and } \\
\text { dermal contact } \\
\text { during } \\
\text { showering }\end{array}$ & $\begin{array}{l}\text { Published upper } \\
\text { bound PFs and } \\
\text { RfDs } \\
\text { Oral RfD used } \\
\text { for TCM }\end{array}$ & $\begin{array}{l}\text { Adaptation of } \\
\text { method after (S. } \\
\text { C. Lee et al. } \\
\text { 2004) } \\
\text { RfD used to } \\
\text { calculate HQ for } \\
\text { TCM as } \\
\text { secondary } \\
\text { carcinogen }\end{array}$ & $\begin{array}{l}\text { MCS (exposure } \\
\text { assessment) }\end{array}$ & $\begin{array}{l}\text { PDFs of } \\
\text { LECR, } \\
\text { annual excess } \\
\text { cases of } \\
\text { cancer and HI } \\
\text { due to } \\
\text { exposure to } \\
\text { THM4 via } \\
\text { showering }\end{array}$ \\
\hline $\begin{array}{l}\text { (Buteau \& } \\
\text { Valcke 2010) }\end{array}$ & $\begin{array}{l}\text { Province of } \\
\text { Quebec, } \\
\text { Canada } \\
\text { (adult } \\
\text { population, } \\
\text { and 2 } \\
\text { presumed } \\
\text { susceptible } \\
\text { subgroups: } \\
\text { toddlers; } \\
\text { infants) }\end{array}$ & $\begin{array}{l}\text { TCM (as } \\
\text { surrogate for } \\
\text { THM4) }\end{array}$ & $\begin{array}{l}\text { Non-cancer } \\
\text { outcomes } \\
\text { (unspecified) }\end{array}$ & $\begin{array}{l}\text { Quarter of } \\
\text { year where } \\
\text { exceedances } \\
\text { of guideline } \\
\text { were more } \\
\text { frequent } \\
\text { and THM } \\
\text { levels } \\
\text { highest } \\
\text { (July- } \\
\text { October). }\end{array}$ & $\begin{array}{l}\text { Two explicit } \\
\text { exposure } \\
\text { scenarios } \\
\text { defined over } \\
\text { high THM } \\
\text { quarter: (1) } \\
\text { average } \\
\text { [TTHM]; (2) } \\
\text { average of all } \\
\text { [TTHM] } \\
>80 \mu \mathrm{g} / \mathrm{L}\end{array}$ & $\begin{array}{l}\text { Multipathway } \\
\text { (ingestion } \\
\text { of DW } \\
\text { [infants } \\
\text { consuming } \\
100 \% \\
\text { formula } \\
\text { mixed with } \\
\text { DW], and } \\
\text { inhalation } \\
\text { and dermal } \\
\text { contact via } \\
\text { showering } \\
\text { [bathing for } \\
\text { infants \& } \\
\text { toddlers] }\end{array}$ & $\begin{array}{l}\text { Distributions fit } \\
\text { to monitoring } \\
\text { data of TCM } \\
\text { concentrations } \\
\text { at tap. } \\
\text { Absorbed doses } \\
\text { calculated for } \\
\text { ingestion, and } \\
\text { for inhalation } \\
\text { and dermal } \\
\text { contact during } \\
\text { showering } \\
\text { using process- } \\
\text { based model }\end{array}$ & $\begin{array}{l}\text { Intermediate } \\
\text { MRLs used as } \\
\text { TRVs to } \\
\text { account for } \\
\text { short exposure } \\
\text { period. MRLs } \\
\text { based on } \\
\text { number of } \\
\text { animal studies } \\
\text { (liver enzyme } \\
\text { activity) and } \\
\text { occupational } \\
\text { studies } \\
\text { (hepatitis) }\end{array}$ & $\begin{array}{l}\text { Method after } \\
\text { (USEPA 2004), } \\
\text { whereby RQ was } \\
\text { calculated for } \\
\text { each exposure } \\
\text { pathway, which } \\
\text { were summed } \\
\text { (since all related } \\
\text { to hepatic effects) }\end{array}$ & $\begin{array}{l}\text { MCS (exposure } \\
\text { assessment) } \\
\text { PDFs of some } \\
\text { exposure variables } \\
\text { and population } \\
\text { characteristics } \\
\text { Sensitivity } \\
\text { analysis for each } \\
\text { input variable }\end{array}$ & $\begin{array}{l}\text { Firstly, } \\
\text { estimation of } \\
\text { non- } \\
\text { carcinogenic } \\
\text { risk (RQ), } \\
\text { then back } \\
\text { calculated to } \\
\text { provide DW } \\
\text { concentration } \\
\text { that should } \\
\text { not be } \\
\text { exceeded to } \\
\text { maintain RQ } \\
<1\end{array}$ \\
\hline $\begin{array}{l}\text { (Chowdhury \& } \\
\text { tall 2010) } \\
\text { RETRACTED }\end{array}$ & $\begin{array}{l}20 \text { most } \\
\text { populated } \\
\text { cities in } \\
\text { Canada }\end{array}$ & THM4 & 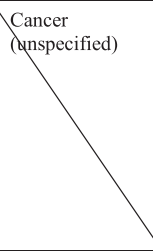 & $\begin{array}{l}\text { Seasonal } \\
\text { dverages for } \\
2002-2008 \\
\text { applied to } \\
\text { lifetipe }\end{array}$ & & $\begin{array}{l}\text { Multipathway } \\
\text { (ingestion } \\
\text { of } \mathrm{DW}, \\
\text { dermal } \\
\text { contadt and } \\
\text { inhalation of } \\
\text { DBPs vid } \\
\text { showering }\end{array}$ & $\begin{array}{l}\text { Pistributions fit } \\
\text { to DW } \\
\text { monitoring data } \\
\text { obtained from } \\
\text { regulatory and } \\
\text { other published } \\
\text { sources }\end{array}$ & $\begin{array}{l}\text { Rublished upper } \\
\text { bound PFs } \\
\text { Oral RfD used } \\
\text { for TCM }\end{array}$ & $\begin{array}{l}\text { Method after (S. } \\
\text { C. Lee et al. } \\
2004) \\
\text { RfD used to } \\
\text { calculate HQ for } \\
\text { TCM as } \\
\text { secondary } \\
\text { carcinogen }\end{array}$ & $\begin{array}{l}\text { MCS (exposure } \\
\text { assessment) } \\
\text { PDFs of } \\
\text { monitoring data - } \\
\text { other expdsure } \\
\text { variables and } \\
\text { population } \\
\text { characteristics } \\
\text { deterministic }\end{array}$ & $\begin{array}{l}\text { LECR and } \\
\text { arnual excess } \\
\text { cases of } \\
\text { cancer due to } \\
\text { multipathway } \\
\text { exposure to } \\
\text { THM4 }\end{array}$ \\
\hline
\end{tabular}


Table 1 (continued)

\begin{tabular}{|c|c|c|c|c|c|c|c|c|c|c|c|}
\hline \multirow[b]{2}{*}{ Reference } & \multicolumn{5}{|c|}{ Issue framing } & \multicolumn{2}{|c|}{ Exposure assessment } & \multirow[b]{2}{*}{$\begin{array}{c}\text { Exposure- } \\
\text { /dose-response }\end{array}$} & \multirow[b]{2}{*}{$\begin{array}{c}\text { Risk } \\
\text { characterisation }\end{array}$} & \multirow[b]{2}{*}{$\begin{array}{l}\text { Analysis of } \\
\text { uncertainty }\end{array}$} & \multirow[b]{2}{*}{$\begin{array}{l}\text { Outcome } \\
\text { metric }\end{array}$} \\
\hline & $\begin{array}{c}\text { Target } \\
\text { population }\end{array}$ & $\begin{array}{c}\text { Hazard/ } \\
\text { agent }\end{array}$ & Health outcome & $\begin{array}{c}\text { Temporal } \\
\text { framing }\end{array}$ & $\begin{array}{l}\text { Scenario(s) } \\
\text { considered }\end{array}$ & $\begin{array}{l}\text { Exposure } \\
\text { route }\end{array}$ & $\begin{array}{c}\text { Exposure } \\
\text { assessment } \\
\text { method }\end{array}$ & & & & \\
\hline $\begin{array}{l}\text { (LaKind et al. } \\
\text { 2010) }\end{array}$ & $\begin{array}{l}\text { NHANES } \\
\text { blood data } \\
\text { (considered } \\
\text { broadly } \\
\text { representative } \\
\text { for US } \\
\text { population) }\end{array}$ & THM4 & $\begin{array}{l}\text { Cancer } \\
\text { (unspecified) } \\
\text { and non-cancer } \\
\text { outcomes } \\
\text { (unspecified) }\end{array}$ & 1999-2004 & None & $\begin{array}{l}\text { Blood levels } \\
\text { of THM from } \\
\text { all exposure } \\
\text { routes }\end{array}$ & $\begin{array}{l}\text { Biomonitoring } \\
\text { equivalents } \\
\text { (BE) calculated } \\
\text { from levels of } \\
\text { THMs in } \\
\sim 2,500 \\
\text { NHANES } \\
\text { blood samples }\end{array}$ & $\begin{array}{l}\text { THM-specific } \\
\text { BEs derived } \\
\text { from blood } \\
\text { concentrations } \\
\text { using } \\
\text { pharmaco- } \\
\text { kinetic human } \\
\text { and animal } \\
\text { data. TCM not } \\
\text { include as PF } \\
\text { withdrawn by } \\
\text { USEPA. }\end{array}$ & $\begin{array}{l}\text { Blood THM } \\
\text { concentrations } \\
\text { compared with } \\
\text { BEs, and each } \\
\text { THM assigned } \\
\text { low, medium or } \\
\text { high priority for } \\
\text { follow-up }\end{array}$ & $\begin{array}{l}95 \% \text { CIs on blood } \\
\text { concentrations } \\
\text { propagated }\end{array}$ & $\begin{array}{l}\text { Priorities for } \\
\text { risk } \\
\text { assessment } \\
\text { follow-up } \\
\text { based on } \\
\text { LECR. } \\
\text { Hazard index } \\
\text { for non- } \\
\text { cancer } \\
\text { outcomes. }\end{array}$ \\
\hline $\begin{array}{l}\text { (Basu et al. } \\
\text { 2011) }\end{array}$ & $\begin{array}{l}10 \text { DWTPs } \\
\text { in } 2 \text { states } \\
\text { (Jharkand } \\
\text { and West } \\
\text { Bengal), } \\
\text { India }\end{array}$ & THM4 & $\begin{array}{l}\text { Cancer } \\
\text { (unspecified) } \\
\text { and non-cancer } \\
\text { outcomes } \\
\text { (unspecified) }\end{array}$ & $\begin{array}{l}\text { March } 2009 \\
\text { to June } \\
2009 ; \\
\text { applied to } \\
\text { lifetime }\end{array}$ & $\begin{array}{l}\text { Worst case } \\
\text { scenario } \\
\text { assumed - } \\
\text { most distal } \\
\text { points in } \\
\text { distribution } \\
\text { system } \\
\text { sampled, in } \\
\text { summer } \\
\text { months }\end{array}$ & $\begin{array}{l}\text { Multipathway } \\
\text { (ingestion } \\
\text { of DW, } \\
\text { dermal } \\
\text { contact and } \\
\text { inhalation of } \\
\text { DBPs via } \\
\text { showering) }\end{array}$ & $\begin{array}{l}44 \text { triplicate tap } \\
\text { water samples } \\
\text { were collected } \\
\text { at DWTP } \\
\text { endpoints and } \\
\text { analyzed from } \\
11 \text { locations }\end{array}$ & $\begin{array}{l}\text { Published upper } \\
\text { bound PFs } \\
\text { (including } \\
\text { rescinded data } \\
\text { for TCM) }\end{array}$ & $\begin{array}{l}\text { Method after (S. } \\
\text { C. Lee et al. } \\
\text { 2004) }\end{array}$ & $\begin{array}{l}\text { Sensitivity } \\
\text { analysis carried } \\
\text { out for some } \\
\text { exposure variables } \\
\text { (exact methods } \\
\text { are unclear) }\end{array}$ & $\begin{array}{l}\text { LECR and HI } \\
\text { due to } \\
\text { multipathway } \\
\text { exposure to } \\
\text { THM4 }\end{array}$ \\
\hline $\begin{array}{l}\text { (Chowdhury et } \\
\text { al. 2011) } \\
\text { In italics: } \\
\text { updates } \\
\text { according to } \\
\text { Chowdhury } \\
\text { (2012) response } \\
\text { to commentary } \\
\text { (Bull et al. 2012) }\end{array}$ & $\begin{array}{l}\text { Canada (all } \\
\text { provinces) }\end{array}$ & THM4 & $\begin{array}{l}\text { Cancer } \\
\text { (unspecified) - } \\
\text { medical costs } \\
\text { calculated } \\
\text { assuming cases } \\
\text { of bladder and } \\
\text { colorectal } \\
\text { cancer }\end{array}$ & $\begin{array}{l}1993 \text { to } \\
2007, \\
\text { depending } \\
\text { on } \\
\text { province; } \\
\text { applied to } \\
\text { lifetime } \\
\text { ( } 70 \mathrm{yrs})\end{array}$ & None & $\begin{array}{l}\text { Multipathway } \\
\text { (ingestion } \\
\text { of DW, } \\
\text { dermal } \\
\text { contact and } \\
\text { inhalation of } \\
\text { DBPs via } \\
\text { showering) }\end{array}$ & $\begin{array}{l}\text { Quarterly } \\
\text { monitoring data } \\
\text { of DW from } \\
\text { household taps } \\
\text { collected from } \\
\text { provincial } \\
\text { offices and } \\
\text { Health Canada } \\
\text { reports. Age- } \\
\text { adjusted intake } \\
\text { rates used. }\end{array}$ & 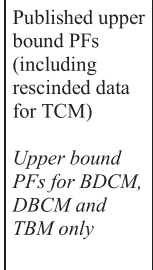 & $\begin{array}{l}\text { Method after (S. } \\
\text { C. Lee et al. } \\
\text { 2004) }\end{array}$ & $\begin{array}{l}\text { MCS }-10^{\text {th }}, 50^{\text {th }} \\
\text { and } 90^{\text {th }} \\
\text { percentiles of } \\
\text { some exposure } \\
\text { variables used to } \\
\text { fit triangular } \\
\text { distributions. }\end{array}$ & $\begin{array}{l}\text { LECR and } \\
\text { annual excess } \\
\text { cases of } \\
\text { cancer, and } \\
\text { healthcare } \\
\text { costs due to } \\
\text { multipathway } \\
\text { exposure to } \\
\text { THM4 }\end{array}$ \\
\hline $\begin{array}{l}\text { (Legay et al. } \\
\text { 2011) }\end{array}$ & $\begin{array}{l}\text { Nine } \\
\text { distributions } \\
\text { systems in } 2 \\
\text { regions of } \\
\text { Quebec, } \\
\text { Canada }\end{array}$ & $\begin{array}{l}\text { THM4 and } \\
\text { DCAA }\end{array}$ & $\begin{array}{l}\text { Cancer } \\
\text { (unspecified) }\end{array}$ & $\begin{array}{l}\text { 2006-2008; } \\
\text { applied to } \\
\text { lifetime }\end{array}$ & $\begin{array}{l}\text { Cancer risk } \\
\text { assessment } \\
\text { considering } \\
\text { chloroform as } \\
\text { a carcinogen } \\
\text { versus } \\
\text { chloroform as } \\
\text { a non- } \\
\text { carcinogen }\end{array}$ & $\begin{array}{l}\text { Multipathway } \\
\text { (ingestion } \\
\text { of DW, } \\
\text { dermal } \\
\text { contact and } \\
\text { inhalation of } \\
\text { DBPs via } \\
\text { showering) }\end{array}$ & $\begin{array}{l}\text { PDFs fit to } \\
\text { monthly sample } \\
\text { survey data } \\
\text { based on DW } \\
\text { sampling from } \\
\text { taps at } 46 \text { sites } \\
\text { (one per zone) } \\
\text { in } 9 \text { distribution } \\
\text { systems }\end{array}$ & $\begin{array}{l}\begin{array}{l}\text { Published upper } \\
\text { bound PFs } \\
\text { (including } \\
\text { rescinded data } \\
\text { on TCM) }\end{array} \\
\text { Separate } \\
\text { analysis carried } \\
\text { out not } \\
\text { including TCM }\end{array}$ & $\begin{array}{l}\text { Method after (S. } \\
\text { C. Lee et al. } \\
\text { 2004) with some } \\
\text { adaptations for } \\
\text { showering model }\end{array}$ & $\begin{array}{l}\text { MCS (exposure } \\
\text { assessment) } \\
\text { PDFs of some } \\
\text { exposure variables } \\
\text { and population } \\
\text { characteristics }\end{array}$ & $\begin{array}{l}\text { LECR due to } \\
\text { multipathway } \\
\text { exposure to } \\
\text { THM4 and } \\
\text { DCAA }\end{array}$ \\
\hline (Liu et al. 2011) & $\begin{array}{l}\text { One DWTP } \\
\text { in Shanghai, } \\
\text { China }\end{array}$ & THM4 & $\begin{array}{l}\text { Cancer } \\
\text { (unspecified) }\end{array}$ & $\begin{array}{l}2009 \\
\text { applied to } \\
\text { lifetime }\end{array}$ & $\begin{array}{l}\text { Seasonal } \\
\text { variations } \\
\text { (spring, } \\
\text { summer, } \\
\text { autumn, } \\
\text { winter) }\end{array}$ & $\begin{array}{l}\text { Multipathway } \\
\text { (ingestion } \\
\text { of DW, } \\
\text { dermal } \\
\text { contact and } \\
\text { inhalation of } \\
\text { DBPs via } \\
\text { showering) }\end{array}$ & $\begin{array}{l}24 \text { samples } \\
\text { taken at DWTP } \\
\text { prior to } \\
\text { distribution }\end{array}$ & $\begin{array}{l}\text { Published upper } \\
\text { bound PFs } \\
\text { (including } \\
\text { rescinded data } \\
\text { for TCM) }\end{array}$ & $\begin{array}{l}\text { Method after (S. } \\
\text { C. Lee et al. } \\
\text { 2004) }\end{array}$ & None & $\begin{array}{l}\text { LECR due to } \\
\text { multipathway } \\
\text { exposure to } \\
\text { THM4 and } \\
\text { DCAA }\end{array}$ \\
\hline $\begin{array}{l}\text { (Pardakhti et al. } \\
\text { 2011) }\end{array}$ & $\begin{array}{l}\text { Seven water } \\
\text { districts in } \\
\text { Tehran, Iran }\end{array}$ & THM4 & $\begin{array}{l}\text { Cancer } \\
\text { (unspecified) }\end{array}$ & $\begin{array}{l}\text { March- } \\
\text { September } \\
2009 ; \\
\text { applied to } \\
\text { lifetime }\end{array}$ & None & $\begin{array}{l}\text { Multipathway } \\
\text { (ingestion } \\
\text { of DW, } \\
\text { dermal } \\
\text { contact and } \\
\text { inhalation of } \\
\text { DBPs via } \\
\text { showering) }\end{array}$ & $\begin{array}{l}63 \text { samples of } \\
\text { household tap } \\
\text { water taken } \\
\text { from } 21 \\
\text { sampling } \\
\text { locations ( } 3 \\
\text { replicate } \\
\text { samples at each } \\
\text { at one time } \\
\text { point) }\end{array}$ & $\begin{array}{l}\text { Published upper } \\
\text { bound PFs } \\
\text { (including } \\
\text { rescinded data } \\
\text { for TCM) and } \\
\text { RfDs }\end{array}$ & $\begin{array}{l}\text { Method after (S. } \\
\text { C. Lee et al. } \\
\text { 2004) }\end{array}$ & None & $\begin{array}{l}\text { LECR and } \\
\text { annual excess } \\
\text { cases of } \\
\text { cancer due to } \\
\text { multipathway } \\
\text { exposure to } \\
\text { THM4 }\end{array}$ \\
\hline $\begin{array}{l}\text { (Yamamoto } \\
\text { 2011) }\end{array}$ & $\begin{array}{l}\text { Three water } \\
\text { utilities } \\
\text { serving } \\
\text { Osaka City, } \\
\text { Japan }\end{array}$ & THM4 & $\begin{array}{l}\text { Cancer } \\
\text { (unspecified) } \\
\text { and non-cancer } \\
\text { outcomes } \\
\text { (unspecified) }\end{array}$ & $\begin{array}{l}\text { Summers } \\
1998-2004, \\
\text { before and } \\
\text { after } \\
\text { installation } \\
\text { of advanced } \\
\text { treatment }\end{array}$ & $\begin{array}{l}\text { Comparison of } \\
\text { conventionally } \\
\text { treated water } \\
\text { and advanced } \\
\text { treated water } \\
\text { (ozonation } \\
\text { step) }\end{array}$ & $\begin{array}{l}\text { Only } \\
\text { ingestion } \\
\text { route was } \\
\text { considered }\end{array}$ & $\begin{array}{l}5 \text { sampling } \\
\text { campaigns of } \\
\text { household tap } \\
\text { water at (48 } \\
\text { measurements } \\
\text { taken at } 31 \\
\text { locations) }\end{array}$ & $\begin{array}{l}\text { Published upper } \\
\text { bound PF ingestion } \\
\text { (including } \\
\text { rescinded data } \\
\text { for TCM) }\end{array}$ & $\begin{array}{l}\text { Based on USEPA } \\
\text { methods for } \\
\text { calculating } \\
\text { lifetime cancer } \\
\text { risk for ingestion }\end{array}$ & $\begin{array}{l}\text { Risks estimated at } \\
\text { mean and minima } \\
\text { and maxima of } \\
\text { exposure } \\
\text { measurements. }\end{array}$ & $\begin{array}{l}\text { LECR, } \\
\text { annual excess } \\
\text { cases of } \\
\text { cancer and HI } \\
\text { due to } \\
\text { multipathway } \\
\text { exposure to } \\
\text { THM4 }\end{array}$ \\
\hline $\begin{array}{l}\text { (Venkataraman } \\
\text { \& Uddameri } \\
\text { 2012) }\end{array}$ & $\begin{array}{l}48 \text { counties } \\
\text { in coastal } \\
\text { Texas, USA, } \\
\text { underlain by } \\
\text { Gulf Coast } \\
\text { aquifer }\end{array}$ & $\begin{array}{l}\text { THM4 used } \\
\text { for showering }\end{array}$ & $\begin{array}{l}\text { Cancer } \\
\text { (unspecified) }\end{array}$ & $\begin{array}{l}\text { 2004-2008; } \\
\text { applied to } \\
\text { lifetime }\end{array}$ & $\begin{array}{l}\text { Inhalation risks } \\
\text { of (a) first } \\
\text { person to use } \\
\text { the shower in } \\
\text { the day; and } \\
\text { (b) subsequent } \\
\text { shower user }\end{array}$ & $\begin{array}{l}\text { Multipathway } \\
\text { (details on } \\
\text { inhalation via } \\
\text { showering; } \\
\text { no details on } \\
\text { ingestion or } \\
\text { dermal } \\
\text { routes) }\end{array}$ & $\begin{array}{l}\text { Predictive } \\
\text { model of THM } \\
\text { formation in } \\
\text { GW using } \\
\text { kriging; } \\
\text { validated } \\
\text { against survey } \\
\text { data of THM4 } \\
\text { for 2004-2008. } \\
\text { Surface water } \\
\text { THM4 data } \\
\text { from } \\
\text { monitoring } \\
\text { survey }\end{array}$ & $\begin{array}{l}\text { Published upper } \\
\text { bound } \mathrm{PF}_{\text {ingestion }} \\
\text { Oral RfD used } \\
\text { for TCM }\end{array}$ & $\begin{array}{l}\text { Based on USEPA } \\
\text { methods for } \\
\text { calculating } \\
\text { lifetime cancer } \\
\text { risk for inhalation } \\
\text { (no details } \\
\text { provided for } \\
\text { ingestion } \\
\text { pathway) } \\
\text { RfD used to } \\
\text { calculate HQ for } \\
\text { TCM as } \\
\text { secondary } \\
\text { carcinogen }\end{array}$ & None & $\begin{array}{l}\text { LECR, and } \\
\text { HI due to } \\
\text { exposure to } \\
\text { THM4 via } \\
\text { showering }\end{array}$ \\
\hline
\end{tabular}


Table 1 (continued)

\begin{tabular}{|c|c|c|c|c|c|c|c|c|c|c|c|}
\hline \multirow{2}{*}{ Reference } & \multicolumn{5}{|c|}{ Issue framing } & \multicolumn{2}{|c|}{ Exposure assessment } & \multirow[b]{2}{*}{$\begin{array}{c}\text { Exposure- } \\
\text { /dose-response }\end{array}$} & \multirow[b]{2}{*}{$\begin{array}{c}\text { Risk } \\
\text { characterisation }\end{array}$} & \multirow[b]{2}{*}{$\begin{array}{l}\text { Analysis of } \\
\text { uncertainty }\end{array}$} & \multirow[b]{2}{*}{$\begin{array}{l}\text { Outcome } \\
\text { metric }\end{array}$} \\
\hline & $\begin{array}{c}\text { Target } \\
\text { population }\end{array}$ & $\begin{array}{c}\text { Hazard/ } \\
\text { agent }\end{array}$ & Health outcome & $\begin{array}{c}\text { Temporal } \\
\text { framing }\end{array}$ & $\begin{array}{l}\text { Scenario(s) } \\
\text { considered }\end{array}$ & $\begin{array}{l}\text { Exposure } \\
\text { route }\end{array}$ & $\begin{array}{c}\text { Exposure } \\
\text { assessment } \\
\text { method }\end{array}$ & & & & \\
\hline $\begin{array}{l}\text { (Amjad et al. } \\
\text { 2013) }\end{array}$ & $\begin{array}{l}\text { Two cities in } \\
\text { Pakistan- } \\
\text { Rawalpindi } \\
\text { and } \\
\text { Islamabad }\end{array}$ & $\begin{array}{l}\text { TCM, BDCM } \\
\text { \& DBCM }\end{array}$ & $\begin{array}{l}\text { Cancer } \\
\text { (unspecified) } \\
\text { and non-cancer } \\
\text { outcomes } \\
\text { (unspecified) }\end{array}$ & $\begin{array}{l}\text { Not } \\
\text { presented }\end{array}$ & None & $\begin{array}{l}\text { Multipathway } \\
\text { (ingestion } \\
\text { of DW, } \\
\text { dermal } \\
\text { contact and } \\
\text { inhalation of } \\
\text { DBPs via } \\
\text { showering) }\end{array}$ & \begin{tabular}{|l|} 
Sampling of tap \\
water at 20 \\
locations
\end{tabular} & $\begin{array}{l}\text { Published upper } \\
\text { bound PFs } \\
\text { (including } \\
\text { rescinded data } \\
\text { for TCM) and } \\
\text { RfDs }\end{array}$ & $\begin{array}{l}\text { Method after (S. } \\
\text { C. Lee et al. } \\
\text { 2004) }\end{array}$ & None & $\begin{array}{l}\text { LECR, and } \\
\text { HI due to } \\
\text { multipathway } \\
\text { exposure to } \\
\text { TCM, BDCM } \\
\text { and DBCM }\end{array}$ \\
\hline (Gan et al. 2013) & $\begin{array}{l}\text { Population } \\
\text { served by } 10 \\
\text { water } \\
\text { treatment } \\
\text { plants in } \\
\text { three } \\
\text { Chinese } \\
\text { cities - } \\
\text { Guangzhou, } \\
\text { Foshan and } \\
\text { Zhuhai }\end{array}$ & $\begin{array}{l}\text { THM4 and } 2 \\
\text { HAAs (DCAA } \\
\& \text { TCAA) }\end{array}$ & $\begin{array}{l}\text { Cancer } \\
\text { (unspecified) }\end{array}$ & $\begin{array}{l}\text { July and } \\
\text { December } \\
2011 \text {, } \\
\text { January and } \\
\text { November } \\
2012 ; \\
\text { applied to } \\
\text { lifetime }\end{array}$ & None & $\begin{array}{l}\text { Multipathway } \\
\text { (ingestion } \\
\text { of DW, } \\
\text { dermal } \\
\text { contact and } \\
\text { inhalation of } \\
\text { DBPs via } \\
\text { showering) }\end{array}$ & \begin{tabular}{|l|} 
Sampling \\
survey of tap \\
water, with \\
calculation of \\
ingestion \\
(drinking only), \\
inhalation and \\
dermal contact \\
(shower stall \\
model) based \\
on mean \\
individual \\
THM \\
concentrations.
\end{tabular} & \begin{tabular}{|l|} 
Published upper \\
bound PFs \\
(including \\
rescinded data \\
for TCM)
\end{tabular} & $\begin{array}{l}\text { Method after (S. } \\
\text { C. Lee et al. } \\
\text { 2004) }\end{array}$ & None & $\begin{array}{l}\text { LECR due to } \\
\text { multipathway } \\
\text { exposure to } \\
\text { THM4 and } 2 \\
\text { HAAs }\end{array}$ \\
\hline (Lee et al. 2013) & $\begin{array}{l}\text { Adult } \\
\text { population } \\
\text { served by } 6 \\
\text { water } \\
\text { treatment } \\
\text { plants in } \\
\text { Seoul, Korea }\end{array}$ & THM4 & $\begin{array}{l}\text { Cancer } \\
\text { (unspecified) }\end{array}$ & $\begin{array}{l}2009 ; \\
\text { applied to } \\
\text { lifetime }\end{array}$ & $\begin{array}{l}\text { Comparison of } \\
\text { boiled water } \\
\text { with unboiled } \\
\text { water for } \\
\text { ingestion } \\
\text { pathway }\end{array}$ & $\begin{array}{l}\text { Multipathway } \\
\text { (ingestion } \\
\text { of DW, } \\
\text { dermal } \\
\text { contact and } \\
\text { inhalation of } \\
\text { DBPs via } \\
\text { showering) }\end{array}$ & $\begin{array}{l}\text { Distributions fit } \\
\text { to monitoring } \\
\text { data of TCM } \\
\text { concentrations } \\
\text { in DW at tap. } \\
\text { Absorbed doses } \\
\text { calculated for } \\
\text { ingestion, and } \\
\text { for inhalation } \\
\text { and dermal } \\
\text { contact during } \\
\text { showering } \\
\text { using process- } \\
\text { based model }\end{array}$ & \begin{tabular}{|l} 
Published upper \\
bound PFs \\
(including \\
rescinded data \\
for TCM)
\end{tabular} & $\begin{array}{l}\text { Adaptation of } \\
\text { method after (S. } \\
\text { C. Lee et al. } \\
\text { 2004) }\end{array}$ & $\begin{array}{l}\text { MCS (exposure } \\
\text { assessment) } \\
\begin{array}{l}\text { Sensitivity } \\
\text { analysis on some } \\
\text { exposure } \\
\text { parameters }\end{array}\end{array}$ & $\begin{array}{l}\text { LECR due to } \\
\text { multipathway } \\
\text { exposure to } \\
\text { THM4 }\end{array}$ \\
\hline $\begin{array}{l}\text { (Karim et al. } \\
\text { 2013) }\end{array}$ & $\begin{array}{l}\text { Population } \\
\text { of } 15 \text { towns } \\
\text { in Karachi, } \\
\text { Pakistan }\end{array}$ & THM4 & $\begin{array}{l}\text { Cancer } \\
\text { (unspecified) } \\
\text { and non-cancer } \\
\text { (unspecified) }\end{array}$ & $\begin{array}{l}\text { March } \\
2010 ; \\
\text { applied to } \\
\text { lifetime }\end{array}$ & N/a & $\begin{array}{l}\text { Ingestion and } \\
\text { dermal } \\
\text { absorption }\end{array}$ & \begin{tabular}{|l|} 
DW samples \\
from 30 \\
locations, \\
interpolation by \\
kriging across \\
entire study \\
area.
\end{tabular} & 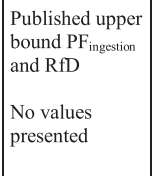 & $\begin{array}{l}\text { Adaptation of } \\
\text { method after (S. } \\
\text { C. Lee et al. } \\
\text { 2004) }\end{array}$ & None & $\begin{array}{l}\text { LECR and HI } \\
\text { due to } \\
\text { multipathway } \\
\text { exposure to } \\
\text { THM4 }\end{array}$ \\
\hline $\begin{array}{l}\text { CDI } \\
\text { LADE } \\
\text { MRL } \\
\text { PF }\end{array}$ & $\begin{array}{l}\text { chronic daily } \\
\text { lifetime aver } \\
\text { minimal risk } \\
\text { potency fact }\end{array}$ & $\begin{array}{l}\text { intake } \\
\text { ge daily exposure } \\
\text { level } \\
\text { r }\end{array}$ & $\begin{array}{l}\text { DALY } \\
\text { LECC } \\
\text { PAH } \\
\text { RQ }\end{array}$ & $\begin{array}{l}\text { ty-adjusted life } \\
\text { excess cases o } \\
\text { omatic hydroca } \\
\text { tient }\end{array}$ & $\begin{array}{l}\text { ar } \\
\text { ancer }\end{array}$ & $\begin{array}{l}\text { DWTP } \\
\text { MCS } \\
\text { PAR\% } \\
\text { TRV }\end{array}$ & $\begin{array}{l}\text { Mrinking water (DV } \\
\text { Monte Carlo simul } \\
\text { Toxulation attribut }\end{array}$ & $\begin{array}{l}\text { W) treatment plant } \\
\text { lation } \\
\text { able risk percent } \\
\text { rence values }\end{array}$ & $\begin{array}{l}\text { GAC } \\
\text { MLE } \\
\text { PDF } \\
\text { VOC }\end{array}$ & $\begin{array}{l}\text { ranulated activated } \\
\text { naximum likelihood } \\
\text { robability distributi } \\
\text { olatile organic com }\end{array}$ & $\begin{array}{l}\text { irbon } \\
\text { stimate } \\
\text { function } \\
\text { und }\end{array}$ \\
\hline
\end{tabular}

provide no information regarding the implications of the burden of cancer on society in terms of type, severity, survival times, population subgroups affected, associated effects on quality of life or costs to healthcare providers. Also, they do not provide useful information on potential health benefits that might be associated with policies or interventions that might alter exposures, either deliberately or as an unintended consequence. Use of data and methods that allow estimation of the burden of specific health outcomes would result in more useful information to a range of decision-makers, as was done in those studies that derived exposure-response data from epidemiological studies. In the Havelaar et al. (2000) study, the maximum likelihood estimate of risk of a specific cancer outcome (renal cell cancer) derived from animal data was explicitly assumed to correspond to the same outcome in humans, and the implications to public health estimated in terms of the severity and duration of that outcome. In one assessment, estimates of LECR were monetised using cost models for bladder and colorectal cancers (Chowdhury et al., 2011), although it later transpired that the methods used in this paper were wrong (Bull et al., 2012). Revised estimates were subsequently published, indicating that the original LECR was considerably overestimated (Chowdhury, 2012). When correctly applied, such an approach might seem to be an improvement over estimates of LECR-based lifetime cancer cases, although the generic nature of LECR limits its validity and we would recommend the more appropriate use of epidemiological data in deriving estimates of attributable cases of a specific cancer outcome. Although epidemiological data on specific health outcomes were used in the USEPA (2005) assessment, estimates of lifetime cancer risk based on slope factors were presented for the purposes of comparison. Notably, these were presented alongside appropriate caveats regarding the limitations associated with use and interpretation of such data.

\subsection{Exposure assessment}

Apart from the laboratory-based, experimental study reviewed (Jo et al., 1990) and study of biomarkers of exposure (LaKind et al., 2010), all studies employed one of three types of exposure assessment: (1) ecological assessments, where populations were assigned exposure levels based on measured or modelled concentrations of DBPs in drinking water; (2) individual level assessment, where a survey of measurements was undertaken for each subject; or (3) binary ecological assessment, where the population was divided according to water supply chlorination status in their area of residence. The first of these methods was used most frequently, with DBP concentrations in drinking water supplies being derived from sampling surveys or monitoring data. In some cases, however, DBP concentrations were entirely based on models, using typical source water quality and treatment procedures as input variables (Black et al., 1996; Havelaar et al., 2000), or on complex semi-empirical models developed using monitoring data and treatment plant characteristics (USEPA, 2005).

Given concentrations of DBPs in drinking water, exposure was calculated either solely for the ingestion pathway, or via multiple pathways. Several process-based models were used to calculate the concentrations of DBPs taken into the body. This multipathway approach is based on various USEPA regulatory risk assessment guidelines (USEPA, 1986, $1999,2002 b$ ) and was introduced into the field of assessing population health impact of DBPs by Lee et al. (2004); many of the subsequent 
studies reviewed used the Lee et al. (2004) study as a template for this kind of assessment.

In those studies where ingestion was considered as the sole pathway of exposure, those pathways in which volatility of DBPs plays an important role were ignored. While this is not necessarily a problem for nonvolatile components of the DBP mixture, it is a serious problem for THMs, since net exposure may in fact be dominated by inhalation during bathing or showering (Nieuwenhuijsen et al., 2000b; Richardson, 2005). In some studies, TCM was used as an indicator for THM4. We would strongly recommend against such an approach: differences in volatilities of individual THMs, in correlations between TCM and the other THMs throughout the distribution network, and in the likely modes of toxicity of individual THMs are all likely to bias risk prediction estimates.

Duration of sampling surveys or periods of monitoring data varied between 2 weeks (Navarro et al., 2007) and 6 years (Williams et al., 2002). In some cases, no information was given regarding the timing or duration of sampling surveys (e.g. Amjad et al. (2013)), which makes the results impossible to interpret. Variations in past exposure were typically ignored. Extrapolation of current levels through time may or may not be valid depending on the temporal stability of contaminant levels in the drinking water sources, methods and technologies employed at treatment plants, and characteristics of the distribution network. When risks based on short term or point estimates of exposure are applied to a population in calculating LECR, for example, those exposure estimates are considered valid averages for 70 years. It has been shown, however, that there is a considerable degree of variability in THM concentrations both within and between 24-hour samples (e.g. Smith et al., 1980; Rodriguez and Serodes, 2001), on a seasonal basis (e.g. Parvez et al., 2011), and among year-on-year averages (e.g. Toledano et al., 2005). Usage habits of water might also be expected to change considerably in time: e.g. tap water consumption to bottled water, bathing to showering, hand washing of clothes and dishes to automatic washing. Calculating health impacts without considering possible changes in exposure over time is particularly problematic for carcinogens, since the bladder cancers associated with exposure to DBPs in epidemiological studies are characterised by long latency times. The USEPA (2005) assessment explicitly incorporated such temporal components in modelling regulatory compliance related to implementing a new rule, and in modelling cessation lag (period between reduction in exposure to a carcinogen and that point when the full risk reduction benefit is realised by affected individuals).

In some studies, concentrations of DBPs at the DWTP were used as exposure estimates for the target population (e.g. Black et al., 1996). DBP concentrations are known to vary considerably within water distribution networks (Amy et al., 2000); levels of THMs have been found to increase with residence time, while other DBPs have been found to decrease (haloacetonitriles, haloketones, chloropicrin, and haloacetic acids) (Chen and Weisel, 1998). Ideally, concentrations of DBPs at the individual consumer taps would be used to characterise target population exposure. Where concentrations at the tap are not available for the entire target population (or are available only at a poor spatial resolution), relationships between concentrations of DBPs at the DWTP and at different points within the distribution network should be explored and quantified.

Geographical information systems (GIS) were used in two studies to produce risk maps based on spatial exposure data. Venkataraman and Uddameri (2012) used TTHM monitoring data and groundwater parameters from a very limited number of locations to model TTHM concentrations at those locations, and kriging was used to interpolate TTHM concentrations across a large area (some 30,000 miles $^{2}$ ). Karim et al. (2013) used kriging of data from a TTHM sampling survey (at 30 locations) to estimate TTHM concentrations for several million inhabitants. As DBP concentrations do not vary smoothly in space, but tend to vary heterogeneously and unpredictably according to the configuration of the network, we consider that neither approach would produce anything other than extremely unreliable exposure estimates for the target populations concerned.

In general, the effort invested in very detailed exposure models in support of an assessment that then uses regulatory tools such as PFs to estimate risk may not be rewarded in terms of proportional improvements in the risk assessment results. In such cases, we would propose that it would be more efficient-and useful from the point of view of informing public health policy-to invest more effort in better characterising the exposure-response functions used. Such risk assessment research should generally be geared towards optimising the use of limited risk management resources (Jardine et al., 2003). In the study by Lee et al. (2004), and several assessments using the same methodology (Tokmak et al., 2004; Viana et al., 2009; Wang et al., 2007a,b), the authors compare cancer risks according to individual trihalomethane species through single exposure pathways. For example, a very detailed process-based model of exposure during showering supports a risk assessment that presents very precise risk estimates under various shower cubicle construction scenarios (Chowdhury and Champagne, 2009). Given the potentially very large uncertainties in the upper bound PFs used to characterise these exposures as risks, quantification of such relationships between behaviour and cancer risk, or comparisons of risks associated with specific exposure routes, are ultimately wholly misleading and have very low utility to decision-makers.

Overall, population health impact assessment should aim to use exposure data that are as similar as possible with exposure as defined in the study (toxicological or epidemiological) from which exposure-response data are derived. This congruence should be sought in terms of exposure medium and routes of intake, and in the temporal and spatial characteristics of those data. In general, the use of animal studies to predict accurately risk in humans is limited because exposure media and routes of intake in animal studies seldom represent those experienced in the human population. For example, high doses of chloroform administered to rodents by gavage as a single daily bolus in corn oil have been associated with tumours, in contrast to administration via drinking water where carcinogenicity has not been demonstrated even at doses 1000 times higher than typical guideline levels (Butterworth and Bogdanffy, 1999; Coffin et al., 2000). However, assessment of exposure in epidemiological studies of DBPs also tends to be limited due to practical difficulties in adequately characterising the many long-term exposure-determining habits and environments of study subjects.

\subsection{Exposure- and dose-response}

The results of population health impact assessments are highly dependent on the data selected to quantify exposure- or dose-response. In many ways, these data are central to the whole assessment, since they dictate the hazard, the form of the exposure metric and the type of health outcome that can be incorporated in the assessment. Where authors do not pay sufficient notice to (a) the framework within which such information was intended to be used; or (b) the veracity and/or validity of this information (i.e. by independently checking the sources from which these data arise), they risk miscalculating their results and may misinterpret the actual risk to the target population.

The majority of studies used toxicological dose-response data (usually upper bound PFs for cancer and RfDs for systemic effects) to estimate risk according to regulatory risk assessment methods. Three studies used epidemiological data in estimating exposure-response functions (Attias et al., 1995; Malcolm et al., 1999; USEPA, 2005). In the Attias et al. (1995) study, epidemiological risk estimates were obtained from a meta-analysis for THM exposure and bladder cancer (Morris et al., 1992) where risk estimates were presented for binary exposure data. In the USEPA (2005) study, OR for binary exposure was derived from a meta-analysis (Villanueva et al., 2003) for bladder cancer. For the purposes of sensitivity analysis, risk estimates for binary exposure data derived from five epidemiological studies and an OR/ 
average TTHM slope from a pooled analysis (Villanueva et al., 2004) were also used. A similar approach was employed for colon and rectal cancer, and for foetal loss. Of the studies using toxicological data, upper bound PFs were most commonly used as they were available from large regulatory risk assessment databases, such as the USEPA IRIS. These upper bound PFs result from application of linearised multistage (LMS) model to animal data so as to extrapolate to effects in humans. Application of LMS in this way generates estimates of nonzero cancer potency even when that parameter is zero (Crump, 1984): as such, actual risk may be anywhere below the quoted upper bound PF and zero (Felter and Dourson, 1998). The USEPA has specifically employed this method in the regulatory context to set guidelines at which the excess risk of cancer is essentially too small to estimate, so that public health is protected. We can say that these upper bound PFs are characterised by "several hidden political components that should not be there, if we wish to claim that risk assessment is a scientific exercise" (Tuomisto, 2005). Those well-acquainted with regulatory risk assessment have never claimed that risks estimated using PFs are accurate, even when differences between animals and humans are disregarded (Hrudey, 2009; Rodricks, 2007). The implications of these political or risk-management decisions, while made explicit in the original literature accompanying the risk assessment (see, for example, Smith, 1996), appear to have been forgotten in many of the cancer risk assessments reviewed here, as it is clear that the use of upper bound PFs will greatly overestimate true cancer risk when applied in population health impact assessment. Some studies used maximum likelihood estimates of cancer potency based on modelling of animal data rather than upper bound estimates so as to reduce the potential for overestimating the risk (Havelaar et al., 2000), and employed probabilistic definitions of cancer potency (Fehr et al., 2003; Havelaar et al., 2000) which were propagated into final risk estimates.

Several studies applied PFs developed for exposure through one pathway of exposure to another (Table 2). For example, $\mathrm{PF}_{\text {ingestion }}$ for a given THM was applied to the inhalation pathway where $\mathrm{PF}_{\text {inhalation }}$ was not available, or for dermal absorption factors. The extrapolation of exposure-route-specific factors from one route to another has been described as "at best difficult and at worst invalid" (Bull et al., 1995). In another study (Black et al., 1996), we found that carcinogenic risks were overestimated for BDCM, as a $\mathrm{PF}_{\text {ingestion was not available and }}$ the PF for DBCM was used instead. Since risks are ultimately summed across pathways and DBPs in this approach to risk assessment, use of inappropriate PFs where specific data are not available necessarily overestimates risk.

All evidence used in an assessment should be valid at the time the assessment is carried out. The contents of online databases such as USEPA IRIS are continually subject to update as new evidence is reviewed. Understanding the validity of data and recognising which data should be used in an assessment are key tasks for the risk assessor. In some of the studies reviewed, outdated PFs were used without justification or explanation (Table 2), a fact that effectively invalidates these results. For example, although TCM was previously considered a genotoxic non-threshold carcinogen, its status has changed considerably in the past two decades as scientific understanding changed, to the extent that employing a PF for TCM after 2001 was not supported by scientific evidence. In 1994, in the absence of better information on the mechanism of its carcinogenicity, the USEPA applied default assumptions of low-dose linearity to TCM, and proposed setting a maximum contaminant level goal (MCLG) of zero. PFs were thus developed for TCM which incorporated this presumptive default, in support of the USEPA's belief that there was no safe level of exposure for chloroform (Pontius, 2003). In 1998, a review of new animal data was carried out which suggested that the available evidence pointed now towards TCM being a threshold carcinogen. Although the MLCG for TCM was not initially revised, an ensuing legal petition resulted in USEPA rescinding its zero MCLG and recommending the use of the RfD in lieu of $\mathrm{PF}_{\text {ingestion. }}$ Cancer effects related to TCM were then thought to be caused by a nongenotoxic cytotoxic mode of action (Butterworth and Bogdanffy, 1999) where hepatotoxicity was a prerequisite for carcinogenesis to occur (King, 2010; USEPA, 2002b). The USEPA IRIS assessment of the cancer risk of TCM formally noted as early as 2001 that the PF had been rescinded because the RfD-based on assessment of liver toxicity-was protective for cancer. All of those studies using a PF for TCM cancer risk after 2001 were carelessly erroneous. The fact that this was done by so many studies would suggest that the reason why a PF should not be used for TCM in cancer risk assessment is widely misunderstood. Risk assessments using linear assumptions about doseresponse of this chemical would grossly overestimate actual risk in human populations (Butterworth, 2005). Although several studies used the RfD of 0.01 in place of the PF $_{\text {ingestion }}$ for TCM by the mid2000s, many authors used the rescinded PFs several years later, even in studies published up to the present day (Fig. 1). Importantly, we noted that this issue of using outdated PFs is not limited to chloroform, but also applies to other THMs. For example, at the time of writing, PF values for BDCM were still available on the IRIS database that will probably be revised in the light of recent toxicological evidence (NTP, 2005), as was done by Health Canada in 2009. As such, we would recommend that authors making any use of data available from sources such as the USEPA IRIS database should check that the online information is truly representative not only of the Agency's actual position, but also of the best current scientific evidence. Publishing studies that neglect to use correct, up-to-date TCM data as issued by the USEPA ultimately undermines the validity of the risk assessment paradigm and may lead to unnecessary public alarm.

The studies that used epidemiological data generally made use of the best human-based information available at the time of their publication. Large, well-conducted epidemiological studies, and meta-analyses and pooled analyses of such data, are an alternative source of data from which exposure-response functions (ERF) can be derived, particularly where there is mechanistic information to support causal interpretation of the reported associations. In the case of epidemiological studies of DBPs and bladder cancer, the importance of recognising the lack of a causal mechanism cannot be overstated. Whereas ERF based on toxicological data can be used to estimate risk of (non-specific) cancer, using data from epidemiological studies allows the estimation of attributable risk of specific health outcomes in the target population, so long as the results are presented alongside a clear description of how well scientific evidence supports causality. Where several studies-or several pooled or meta-analyses-exist, sensitivity analysis should be carried out to quantify the potential impacts of their use on assessment results.

Interpretation of the results of epidemiological studies on DBPs and cancer-and their use in risk assessments-requires an understanding and acceptance of the various uncertainties that influence them. Measurement error in exposure assessment is a major concern, particularly since retrospective estimation of long-term exposure is difficult in the absence of reliable monitoring data. Since almost all studies have used a case-control design in order to be powerful enough to detect modest effects, there is a potential for recall bias. Several of these studies may also be subject to bias due to the use of hospital-based controls, where selection of diseases may not be accurate. In addition, the inaccuracies present in exposure estimates in these studies are indeed limited by non-systematic biases that may lead to reduction in precision of risk estimates, but the lack of specificity in the exposure metrics used also confers some advantage when ERFs derived from those studies are applied in population health impact assessment. Since the putative agents among the > 600 DBPs in water have not been identified, existing epidemiological studies have tended to employ relatively simple proxies of exposure, which may be prone to misclassification of true exposure. This is evidently a weakness of existing epidemiological studies, but concentrations of THMs in the drinking water have nonetheless served as markers for unknown putative agents, and consistency in the risk estimates for bladder cancer may demonstrate that use of such a surrogate is in some way indicative of true exposure, and foregoes the need fully 
Table 2

Summary of dose/exposure-response functions employed.

\begin{tabular}{|c|c|}
\hline Name of study & PFs/SFs/RfDs (pathway, value, citation) \\
\hline Lahey and Connor (1983) & $\begin{array}{l}\text { TCM } \\
\text { - PF ingestion used for oral intake } \\
\text { - Value not presented } \\
\text { - Source of data not presented }\end{array}$ \\
\hline Jo et al. (1990) & $\begin{array}{l}\text { TCM } \\
\text { - } \mathrm{PF}_{\text {ingestion }} \text { used for oral intake, inhalation and dermal contact } \\
\text { - TCM }=0.26 \text { (mg/kg-day) })^{-1} \\
\text { - Published PF (upper } 95 \% \mathrm{CI} \text { ) derived from linearised model applied to single ingestion animal study }\end{array}$ \\
\hline Attias et al. (1995) & $\begin{array}{l}\text { THM4 } \\
\text { - Unit risk used for oral intake } \\
\text { - } \mathrm{TCM}=0.00000027 ; \mathrm{BDCM}=0.0000046 ; \mathrm{DBCM}=0.00000304 ; \mathrm{TBM}=0.000000304 \text { (units of risk per } 1 \mu \mathrm{g} / \mathrm{L} \text { ) } \\
\text { - Unit risks (upper } 95 \% \mathrm{CI} \text { ) estimated from toxicological data using multistage and linearised multistage models }\end{array}$ \\
\hline Black et al. (1996) & $\begin{array}{l}\text { THM4 } \\
\text { - } F_{\text {ingestion }} \text { used for oral intake } \\
\text { - } \text { Published PF (upper 95\%): TCM, BDCM and TBM from USEPA (1994); DBCM assumed equivalent to BDCM } \\
\text { HAAs (DCAA and TCAA) } \\
\text { - PF } \text { ingestion used for ingestion pathway } \\
\text { - DCAA = 0.11; TCAA = 0.083 (units of cases/person/lifetime/(mg/kg body weight/dday)) } \\
\text { - PFs from Bull and Kopfler (1991) }\end{array}$ \\
\hline Chung et al. (1997) & $\begin{array}{l}\text { THM4 } \\
\text { - Single pathway (presumably ingestion) PF used for oral intake, inhalation and dermal contact } \\
\text { - TCM }=0.00000096 ; \mathrm{BDCM}=0.00000415 ; \mathrm{DBCM}=0.0000028 ; \mathrm{TBM}=0.000000415 \text { (units of }(\mu \mathrm{g} / \mathrm{L})^{-1} \text { ) } \\
\text { - Unit risks ( } 95 \% \text { upper bound) obtained using TOX-RISK package (multistage model fitted to animal data) }\end{array}$ \\
\hline Malcolm et al. (1999) & Epidemiological data used \\
\hline Havelaar et al. (2000) & $\begin{array}{l}\text { Bromate } \\
\text { - Ingestion dose-response data applied to oral intake } \\
\text { - Point estimate for dose-response parameter for bromate }=0.0030 \mathrm{~kg}^{2 / 3} / \mathrm{mg} \text { (units of renal cell cancer induction in rats per mg } \mathrm{KBrO}_{3} /(\mathrm{kg} \\
\left.\text { body weight } \mathrm{kg}^{2 / 3}\right) \text { ) } \\
\text { - Dose-response fitted by two-stage model based on three published studies }\end{array}$ \\
\hline Hsu et al. (2001) & $\begin{array}{l}\text { THM4 } \\
\text { - } \mathrm{PF}_{\text {ingestion }} \text { used for oral intake } \\
\cdot \mathrm{TCM}=0.0061 ; \mathrm{BDCM}=0.062 ; \mathrm{DBCM}=0.0084 ; \mathrm{TBM}=0.0079 \text { (units of risk per } \mathrm{mg} / \mathrm{kg} / \mathrm{day} \text { ) } \\
\text { - PFs ( } 95 \% \text { upper bound) for all THM4 cited as USEPA (1999) }\end{array}$ \\
\hline Sadiq et al. (2002) & $\begin{array}{l}\text { TCM } \\
\text { - PF } \text { ingestion used for oral intake } \\
\text { - } 0.0061 \text { (units of } \mathrm{mg} / \mathrm{kg} / \mathrm{day}) \\
\text { - PF (95\% upper bound) from IRIS database, cited as "USEPA (2001)" }\end{array}$ \\
\hline Williams et al. (2002) & $\begin{array}{l}\text { TCM } \\
\text { - } \mathrm{PF}_{\text {ingestion }} \text { used for oral pathway and dermal contact; } \mathrm{PF}_{\text {inhalation }} \text { used for inhalation } \\
\text { - } \mathrm{PF}_{\text {ingestion }}=0.0031 ; \mathrm{PF}_{\text {inhalation }}=0.0019 \text { (units of } \mathrm{mg} \mathrm{kg}^{-1} \mathrm{day}^{-1} \text { ) } \\
\text { - PFs from California EPA, cited as "OEHHA (2010)" }\end{array}$ \\
\hline Fehr et al. (2003) & $\begin{array}{l}\text { TCM and BDCM } \\
\text { - PF } \text { ingestion used for oral intake } \\
\text { - PF values not presented } \\
\text { - PF was specified as a) a 95\% upper bound point estimate and b) a PDF } \\
\text { - PF from WHO guidelines cited as "WHO (1993) and WHO (1998)" }\end{array}$ \\
\hline Sofuoglu et al. (2003) & $\begin{array}{l}\text { TCM } \\
\text { • PF (values not presented in paper) from IRIS database, cited as "USEPA (2001)" }\end{array}$ \\
\hline Lee et al. (2004) & 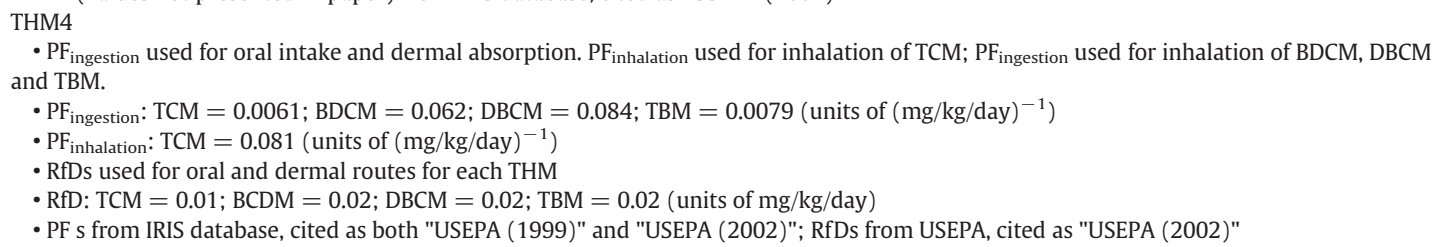 \\
\hline Tokmak et al. (2004) & $\begin{array}{l}\text { THM4 } \\
\text { - Methodology of Lee et al. (2004) is cited as the source of the cancer risk assessment methodology used, but no values for PFs or RfDs are } \\
\text { presented, nor which PFs were used for specific pathways etc. } \\
\text { • Authors confirmed that the same PFs and RfDs were used as in Lee et al. (2004) }\end{array}$ \\
\hline USEPA (2005) & Epidemiological data used \\
\hline Kavcar et al. (2006) & $\begin{array}{l}\text { THM4 } \\
\text { - PF } F_{\text {ingestion used for oral pathway }} \\
\text { - TCM }=0.01(\mathrm{mg} / \mathrm{kg} / \text { day })^{-1}\left(\mathrm{RfD} \text { used instead of withdrawn } \mathrm{PF}_{\text {ingestion }}\right) \text {; values of } \mathrm{PF}_{\text {ingestion }} \text { and RfD for BDCM, DBCM and TBM not presented } \\
\text { - All PFs and RfD from IRIS database, cited as "IRIS (2005)" }\end{array}$ \\
\hline Nazir and Khan (2006) & $\begin{array}{l}\text { TCM } \\
\text { - RfD used for oral pathway (recognised as having replaced } \mathrm{PF}_{\text {ingestion }} \text { ); however, } \mathrm{PF}_{\text {ingestion }} \text { used for dermal contact and inhalation } \\
\text { • RfD }=0.01 \mathrm{mg} / \mathrm{kg} \text {-day; } \mathrm{PF}_{\text {ingestion }}=0.0061(\mathrm{mg} / \mathrm{kg}-\text { day })^{-1} \text { for dermal and inhalation pathways } \\
\text { - IRIS database, cited as both "US Environmental Protection Agency (1999)" and "US Environmental Protection Agency (2001)" }\end{array}$ \\
\hline Uyak (2006) & $\begin{array}{l}\text { THM4 } \\
\text { - } \mathrm{PF}_{\text {ingestion }} \text { used for oral pathway and dermal contact; } \mathrm{PF}_{\text {inhalation }} \text { used for inhalation pathway (TCM only) } \\
\text { - } \mathrm{PF}_{\text {ingestion }}: \mathrm{TCM}=0.0061 ; \mathrm{BDCM}=0.062 ; \mathrm{DBCM}=0.084 ; \mathrm{TBM}=0.0079 \text { (units of }(\mathrm{mg} / \mathrm{kg} / \mathrm{day})^{-1} \text { ) } \\
\text { - } \mathrm{PF} \text { inhalation } \mathrm{TCM}=0.081 \text { (units of }(\mathrm{mg} / \mathrm{kg} / \mathrm{day})^{-1} \text { ) } \\
\text { - RfD: TCM }=0.01 ; \mathrm{BDCM}=0.02 ; \mathrm{DBCM}=0.02 ; \mathrm{TBM}=0.02 \text { (units of } \mathrm{mg} / \mathrm{kg} / \mathrm{day}) \\
\text { - PFs and RfDs are identical to those in Lee et al. (2004); source cited as "USEPA (1999)" }\end{array}$ \\
\hline
\end{tabular}


Table 2 (continued)

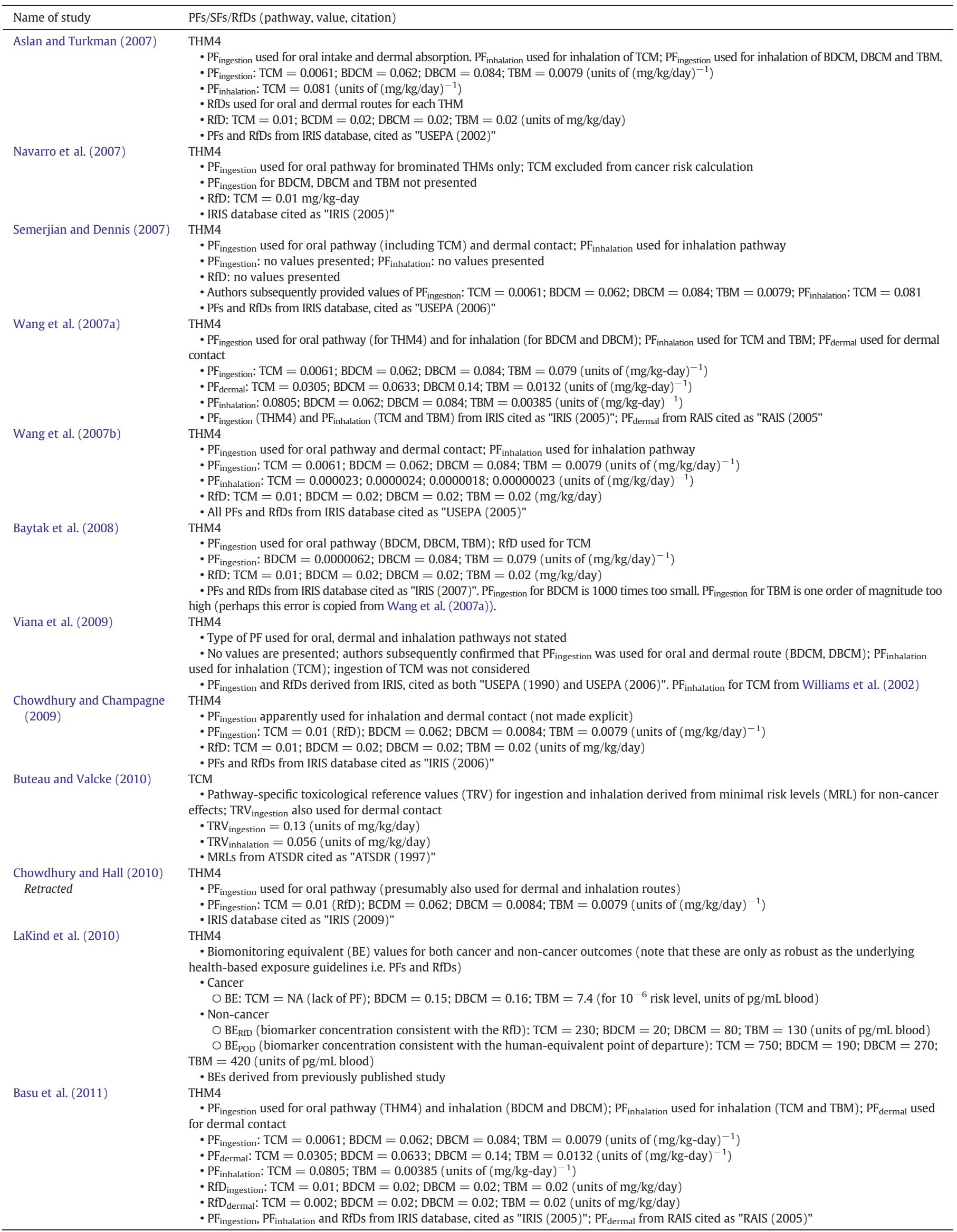


Table 2 (continued)

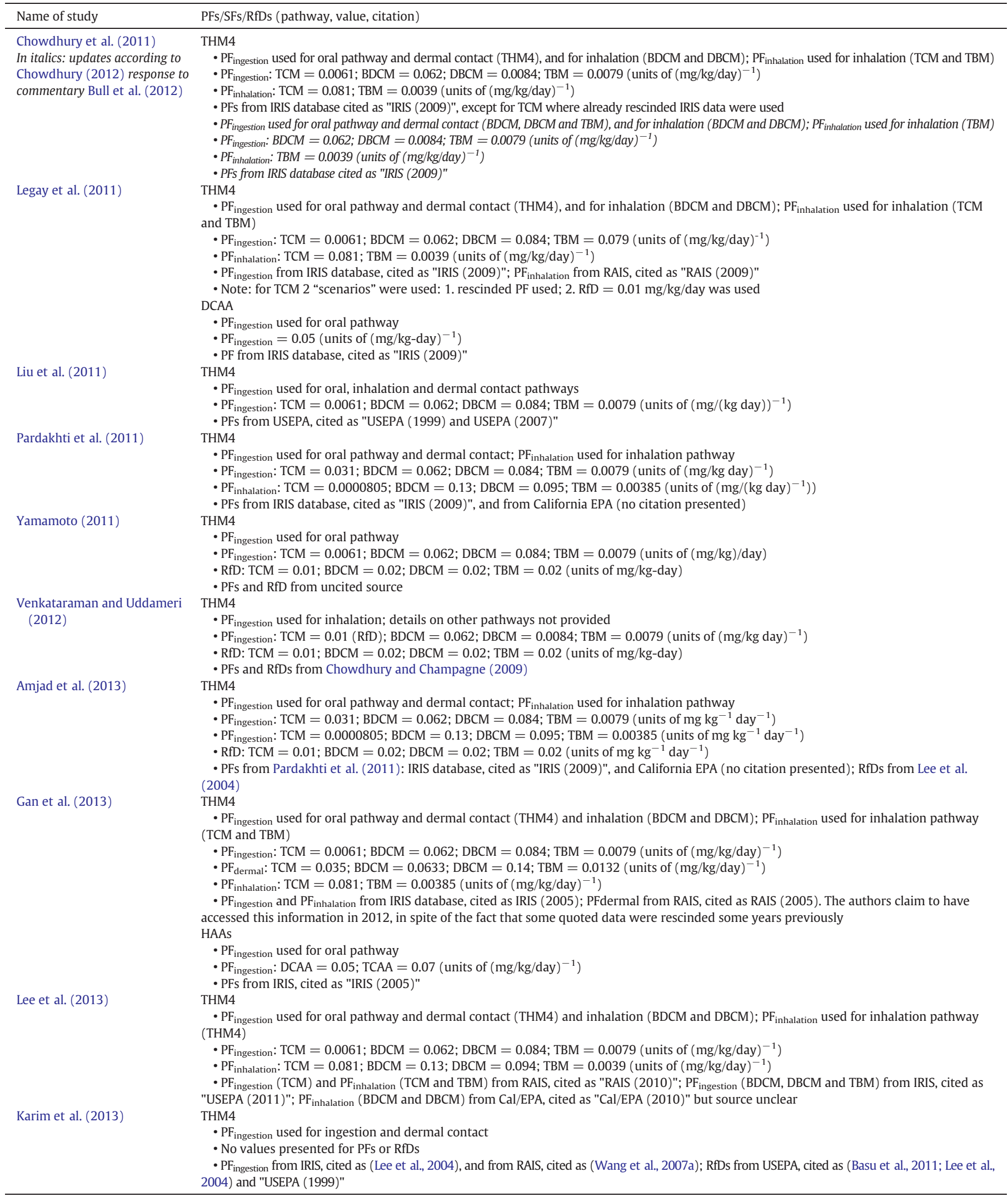

to characterise exposure through all possible pathways to all possible DBPs, which would be prohibitively resource-intensive. Importantly, estimates of LECR calculated from toxicological data on THMs alone have been shown to be some two orders of magnitude lower than those calculated from epidemiological data, where THM concentrations can be considered a surrogate for an unknown putative agent (Bull, 2012). 
Where age- and sex-stratified ERFs can be derived from epidemiological data, these should be applied to the respective strata within the target population.

In spite of the results of the assessments depending heavily on the ERF data used, key information was omitted from a number of reports (Table 2). Specific PFs used in calculating carcinogenic risks were not reported in 8 studies. In several studies, PFs were quoted that would not have been in agreement with cited databases at the time of publication, or sources of data were cited so vaguely as to make verification of PFs impossible. In a few studies, no citation was provided whatsoever for these data. In other cases, the values of PFs reported (and potentially used) were incorrect. In one case, $\mathrm{PF}_{\text {ingestion }}$ for TBM was reported an order of magnitude too high (Wang et al., 2007a) - this error appears to have been reproduced afterwards in another paper (Baytak et al., 2008). The value reported in one paper for $\mathrm{PF}_{\text {ingestion }}$ for $\mathrm{DBCM}$ was an order of magnitude too low (Chowdhury and Champagne, 2009), an error that was apparently propagated into two subsequent papers (Chowdhury and Hall, 2010 (retracted); Chowdhury et al., 2011). In fact, the Chowdhury \& Hall paper was retracted specifically because the IRIS RfD was erroneously used as though it were a PF (thereby completely invalidating the results), and the authors did not publish an erratum when these errors were identified. By graphically representing the $\mathrm{PF}_{\text {ingestion }}$ from all studies (Fig. 1), it is possible to see that the same values were used by many studies over a period of 2 decades, sometimes without paying heed to updated advice regarding the use of these data. There is considerably more variation over time among the PFs used for dermal (Fig. 2) and inhalation (Fig. 3) exposure routes, perhaps partly because in studies seeking to include risk estimation for exposure DBCM and BDCM by inhalation, the authors either sought alternative data when the USEPA ceased to support their use, or continued using them regardless. The central role that dose- and exposure-response data play in population health impact assessment should not be underestimated: these data should be included in the reporting of assessments, and unambiguous citations (including the date of access in the case of online databases) provided to the sources from which they have been obtained. Full review of the validity and suitability of these data should be carried out before using them to characterise risk in a target population.

\subsection{Risk characterisation}

With some notable exceptions, the overwhelming majority of studies focused on estimation of cancer risks associated with DBP exposure, as might be expected given societal concerns about DBPs as possible human carcinogens. Lifetime excess risk of cancer was characterised in most studies by multiplying exposures by PFs to give lifetime cancer risks (after methodology in, for example, Anderson and USEPA Carcinogen Assessment Group, 1983; USEPA, 1986; Smith, 1996). For non-cancer outcomes, exposure was divided by RfDs to yield $\mathrm{HQ}$ (or their sum across pathways, hazard index (HI)). Several of the studies looking at LECR proceeded to calculate the number of lifetime excess cancer cases in a population by multiplying the estimate of LECR by the target population of interest.

Three studies used epidemiological data to assess risks in a population related to exposure to DBPs. One of these (Attias et al., 1995) estimated the incremental lifetime carcinogenic risk (or unit risk), for a hypothetical human population subjected to $1 \mu \mathrm{g} / \mathrm{L}$ of TTHM. This was subsequently used in the same way as a PF to predict the LECR in a population based on its exposure. The second study estimated the cases of disease (cancers of the bladder, colon and rectum, and birth defects)

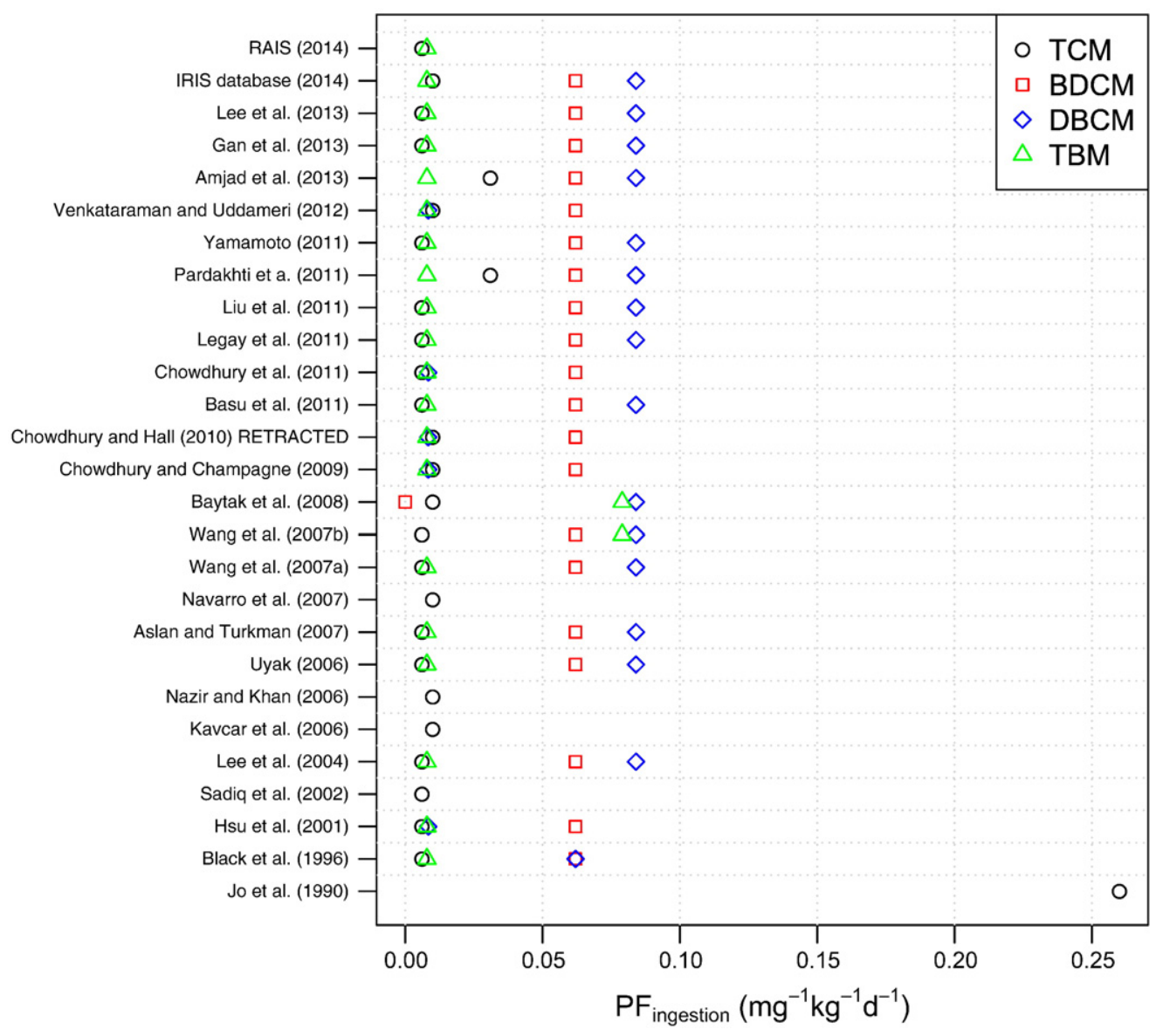

Fig. 1. Cancer potency factors for trihalomethanes used for ingestion route, by study (presented chronologically). 


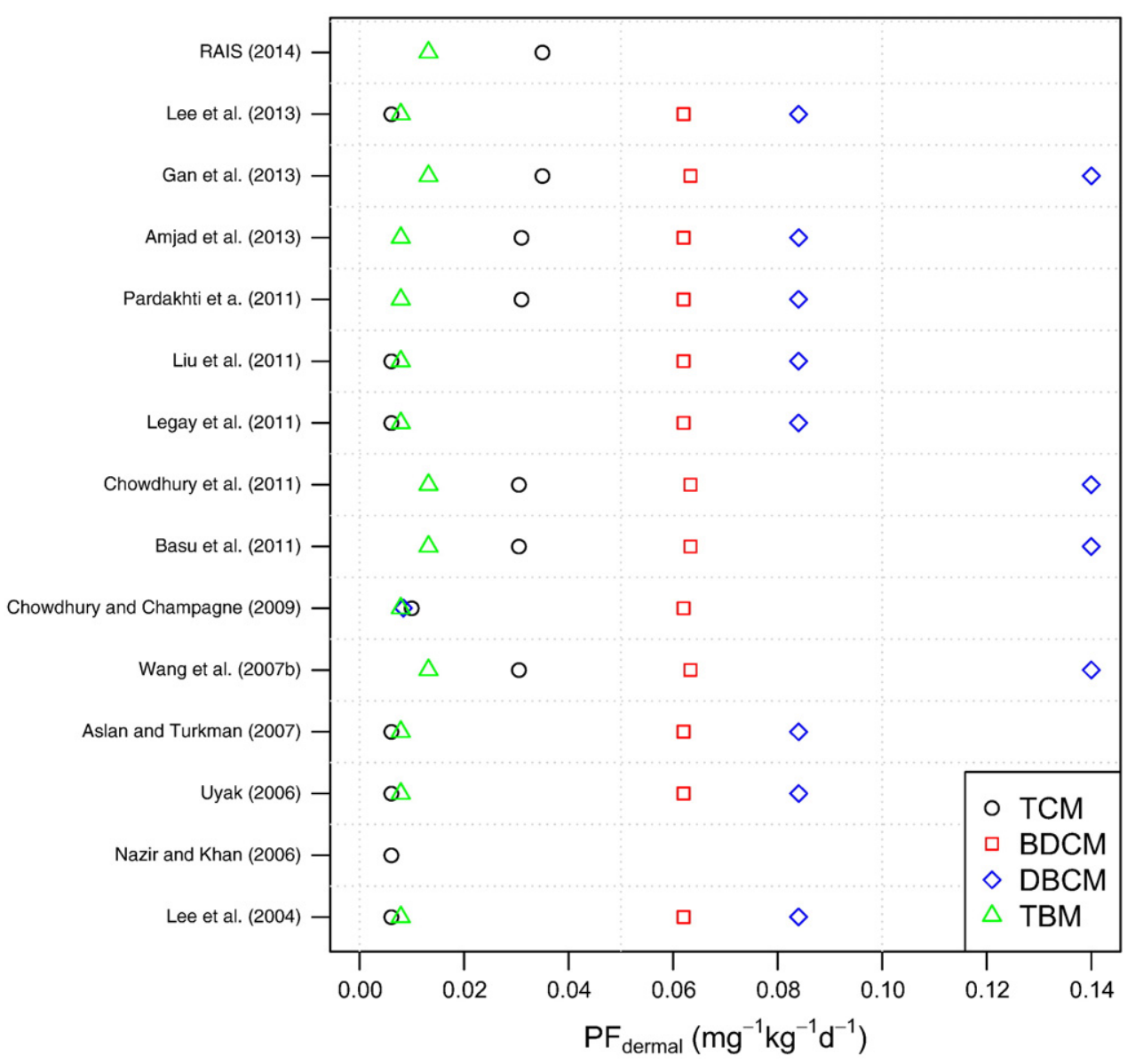

Fig. 2. Cancer potency factors for trihalomethanes used for dermal route, by study (presented chronologically).

attributable to DBP exposure (Malcolm et al., 1999) using population attributable risk percent (PAR\%) using the formula:

$P A R \%=\left[\frac{P e(R R-1)}{1+P e(R R-1)}\right] \cdot 100$

where Pe is the proportion of the population exposed, and $R R$ is the relative risk from an epidemiological study. The result was then applied to cancer registry and birth defect registry data for one year, and reported as the annual number of cases attributable to DBP contamination of drinking water. The third study (USEPA, 2005) calculated the PAR associated with exposure to DBPs and subsequently number of annual cases of bladder cancer that would be avoided after the entire population would have been exposed for a lifetime under new exposure conditions. In the one study that considered risks of microbial disease (Havelaar et al., 2000), infection from C. parvum was estimated using an exponential dose-response model based on volunteer data. Daily risks were cumulated over a year to provide an annual risk for the population. In order to compare between two very different kinds of disease endpoints, namely renal cell cancer and microbial disease, Havelaar et al. (2000) transformed estimates of cases of disease into disability-adjusted life years (DALYs) using clinical data relating to the severity and duration the diseases in question.

There are a number of clear advantages associated with calculating attributable risks or cases of disease using human exposure-response data. Firstly, there is no uncertainty introduced through extrapolating between species, though there are, of course, limitations to the transferability of an ERF based on epidemiological data, in particular due to differences in other factors contributing to disease (genetics or behaviours, for example), between the population from which the ERF was obtained and the target population of the assessment. Secondly, attributable cases of specific disease endpoints can be predicted in the target population, which is of considerable value to decision-makers. Thirdly, there is less of a constraint to focus on individual chemicals, for which toxicological data may be scarce, or of limited applicability to humans; exposure proxies used in the epidemiological studies may be considered to reflect overall exposure to DBPs (albeit with associated loss of precision in the risk estimates), allowing us to forego assumptions regarding the putative agent or agents in the DBP mixture.

In a number of the studies calculating LECR, upper bound risk estimates were further projected onto either hypothetical populations (Black et al., 1996; Havelaar et al., 2000; Jo et al., 1990) or, most commonly, onto the specific population of an area, region or country experiencing a particular exposure, in order to arrive at an estimate of the excess cases of cancer that could be expected in such a population in a year, or over a lifetime. One major weakness to this approach lies in the multifactorial nature of carcinogenesis, with rates for most cancers increasing dramatically with age. Given that the age structure of those target populations is not taken into account, comparisons of cases of cancer calculated in this way across different areas are likely to present a very distorted message to decision-makers. Estimates of the attributable cases of specific types cancer were presented in those studies using epidemiological data to quantify exposure-response (Attias et al., 1995; Malcolm et al., 1999; USEPA, 2005) and as such this approach ostensibly ought to provide more specific and meaningful information to decision makers. However, the estimates of preventable cancer deaths in New Zealand in 1995 published by Malcolm et al. (1999) are approximately twice as high as our own estimates for the New Zealand population in 2012 (not presented), which we calculated using relative risks from the only meta-analysis available at the time, 


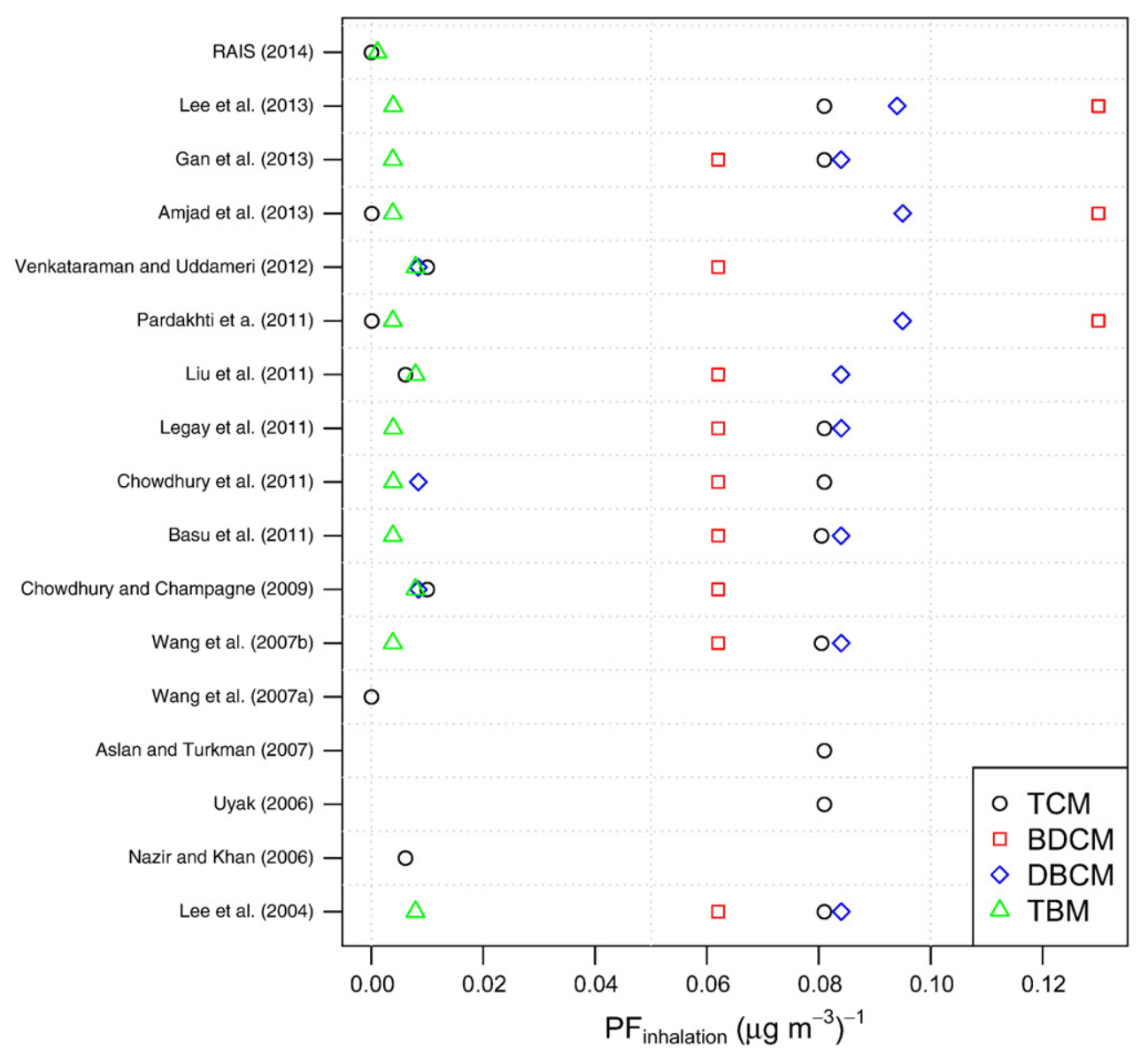

Fig. 3. Cancer potency factors for trihalomethanes used for inhalation route, by study (presented chronologically).

namely that of Morris et al. (1992). This apparent overestimation may be due to improvements in survival for bladder and colorectal cancers between 1995 and 2012. However, our own estimates of attributable burden due to colorectal cancer had wide 95\% uncertainty intervals that included zero and for this reason emphasise that estimates of $\mathrm{AF}_{\mathrm{P}}$ and attributable cases be presented with their associated uncertainty intervals. It is unclear how unit risks were calculated from relative risks in the Attias et al. (1995) study, and it is not explained in that study why more easily interpretable estimates of attributable fractions, for example, were not calculated instead.

Typically the temporal nature of a population health impact assessment is determined by time-related characteristics of the exposure data. In some cases, demographic or health data may also be used to define this temporal scope. In one study, baseline rates of bladder cancer were averaged over several decades, thereby defining the timescale of the assessment and implicitly accounting for latency of effects (Attias et al., 1995). The means by which latency and temporality were explicitly considered in the USEPA (2005) study discussed above represent an example of how to treat these issues. Some assessments that used ERFs derived from epidemiological data were purely cross-sectional in their approach, in spite of the fact that they were addressing cancers known to be characterised by long latency. In studies estimating lifetime cancer risk, a lifetime of exposure was generally fixed at 70 years; where lifetime cases of cancer were estimated for specific populations, it is hard to know exactly to which population such estimates should be assigned. For example, where a target population is assigned a LECR, this should be understood to be the number of excess cancer cases estimated for a population of individuals, each living for 70 years, irrespective of their current age i.e. for those of 70 years of age and older, this risk is presented for exposure in the past, for those of 69 years of age and under, the risk is presented for exposure yet to occur. Interpreting such a metric is far from straightforward given the uncertainties in past and future exposures, and limits the usefulness of such an approach. Also, this method takes no account for increases in cancer risk with increasing age. The effect of not explicitly determining the timeframe of an assessment may result in estimates of exposure and associated risk being over- or underestimated unpredictably. In general, when estimating the attributable cases of a disease in a target population, age- and sex-stratified data should be used, for example:

Attributable cases $=\sum_{\mathrm{i}, \mathrm{j}}\left[\frac{P e_{i j}\left(R R_{i j}-1\right)}{1+P e_{i j}\left(R R_{i j}-1\right)}\right] \cdot \frac{I_{i j}}{100,000} \cdot N_{i j}$

where $P e_{i j}$ is the proportion of the target population exposed in $i$ age groups and for $j$ sexes, $R R_{i j}$ is the age- and sex-stratified relative risk, $I_{i j}$ is the annual incidence of the disease per 100,000 of the target population, and $N_{i j}$ is the age- and sex-stratified number of people in the target population.

Cumulative exposure and latency associated with chronic health effects (e.g. time between first exposure and development of a cancer) present themselves as complex issues to the assessor. While crosssectional modes are static, in the sense that they use current exposure and health data to estimate a burden of disease attributable to a cancer, this is not robust from a temporal standpoint. Such models do not account for the fact that cancer prevalence rates at the current time reflect past exposure to contaminants. Historic exposure data are seldom available for drinking water contaminants due to the relatively recent introduction of monitoring systems. The assessor is then faced with a tradeoff between introducing uncertainties through extrapolating exposure 
into the past, and extrapolating exposure and prevalence data into the future. Historic exposure to drinking water contaminants has been estimated in epidemiologic studies by using historical data on treatment type and source water to build scenarios about levels of DBPs in the past (e.g. Villanueva et al., 2004); similar approaches could be used to estimate the population health impacts under baseline conditions. Conversely, scenarios can be employed to account for the health impacts of specific decisions made today, for example regarding treatment technologies. In either case, it is important to specify precisely the target populations for which health impacts are being assessed, as well as the period of exposure and latency periods to which these relate.

Taking account of various aspects of time in population health impact assessments requires consideration of duration, frequency and temporal variability of exposure, as well as time elapsed between exposure and development of disease (latency). Where data or methods are insufficient for a thorough consideration of timing, assumptions and their associated limitations should be described. In most of the studies reviewed, the temporal nature was generally not stated explicitly, and time-related limitations were not discussed.

\subsection{Scenarios}

Some studies explicitly defined scenarios under which risk estimates might be compared in support of decision-making. These scenarios included different waste disposal policies (Lahey and Connor, 1983), implementation of carbon removal technologies or alternative water treatment measures (Black et al., 1996; Sadiq et al., 2002; USEPA, 2005; Wang et al., 2007b; Yamamoto, 2011), water privatisation policies (Fehr et al., 2003), and chlorination versus no chlorination (Malcolm et al., 1999). Other studies compared risks under alternative exposure scenarios, such as showering versus drinking (Jo et al., 1990), shower design parameters (Chowdhury and Champagne, 2009), shower usage (Nazir and Khan, 2006; Venkataraman and Uddameri, 2012), and consumption of bottled water versus tap water (Kavcar et al., 2006). Others compared risks related to different kinds of exposure-response data (Attias et al., 1995), or under different assumptions regarding the carcinogenicity of chloroform (Legay et al., 2011). Only one study compared the competing risks associated with disinfection: i.e. risks associated with microbial contamination and with DBPs (Havelaar et al., 2000). Multiple scenarios relating to changes in regulations and compliance were compared with three separate sets of baseline conditions in the USEPA (2005) study. Those studies that did not explicitly define scenarios presented estimates of risk associated with DBP exposure versus implied counterfactual scenarios of zero risk at zero exposure. This approach misses the opportunity of supporting relevant decision-making processes; more fundamentally, however, the implicit scenario of zero DBPs is neither plausible nor particularly useful where chemical disinfection is nearubiquitous and serves an important purpose in controlling microbial contamination. Drastic, all-or-nothing scenarios do little to inform policy if they are not realistic.

Ideally, assessors should be guided by discussion with decisionmakers so that scenarios represent actual or (realistic) potential policy questions. We were surprised to find only one study that weighed up the risks (DBPs) and benefits (drinking water free of pathogens) associated with disinfection (Havelaar et al., 2000), given the importance of such a risk-benefit analysis and the very direct implications of such research to policymakers and drinking water providers. In the most extensive study reviewed, considerations relating to microbial quality were accounted for in separate assessments used to develop the regulatory scenarios employed in the DBP assessment which did not pose microbial risks to public health (USEPA, 2005). We would argue that modelling potential effects on health of other hypothetical scenarios relating to DBPs-for example, irrespective of whether they are societally relevant or politically, technically or economically possible-is ultimately an inefficient use of scientific resources; identifying the most relevant policy questions and building realistic scenarios should form a key part of the scoping phase of any population health impact assessment. Narrowing the scope of a population health impact assessment to a single contaminant and presenting a risk only against an implicit counterfactual scenario of zero exposure may lead to inappropriate risk management decisions. The value of population health impact assessments should be seen in terms of better understanding the multiple risks in drinking water disinfection and supply so that decisions and policies might be made for the overall improvement of public health.

\subsection{Analysis of uncertainty}

In the context of regulatory risk assessment the use of default values and assumptions is a necessary means of achieving consistency among evaluations of different hazards. Many of the studies reviewed had used data based on similar default values and assumptions in spite of their use outside of the regulatory sphere. Such studies may produce biased results, and may underestimate uncertainty introduced, for example, for true variability in characteristics, behaviours and structure of the target population. These studies would have gained by better characterising such uncertainties; at the very least many would have benefitted from documenting possible sources of uncertainty unaccounted for and the limitations that these would impose on their results. Thorough lists of modelling assumptions, the uncertainties associated with them, and their potential effects on results were presented in very few studies.

Studies which included some analysis of uncertainty used a variety of methods, ranging from sensitivity analysis employing different values of PF, to probabilistic modelling and comprehensive sensitivity analyses of stochastic, parametric, model and data uncertainties. The USEPA (2005) study featured by far the most comprehensive approach to treat uncertainties-incorporating modelling, simulations, extensive sensitivity analyses among other approaches-a discussion of which is outside of the scope of this paper.

The influence of using different data sets, methods or models on results was investigated in some studies, by presentation of systematic univariate sensitivity analysis, or, in the majority of studies by using ad hoc methods of sensitivity analysis. In one study, exposure parameters (some of which were defined probabilistically) were systematically increased by $10 \%$ and the effect on the total absorbed dose was calculated (Buteau and Valcke, 2010). Although this approach yielded useful information regarding the system being modelled and the parts of that system on which more attention should be focused with regard to accuracy and precision, no attempt was made to assess the sensitivity of the overall assessment results to potential uncertainties in the ERF, thereby missing potentially important sources of uncertainty that might completely alter the findings of the study. Focusing all attention on the exposure side of the causal chain that is drawn between hazard, exposure and health risk-and failing to recognise the potential significance of uncertainties in the ERF in this chain-represents a serious oversight.

Probabilistic simulation techniques such as Monte Carlo analysis were used to characterise uncertainty in exposure assessment in a number of studies (see Table 1). Deterministic modelling is increasingly considered as an inappropriate means of assessing uncertain risks (Frey, 1992; Reckhow, 1994; Sander et al., 2006). Employing probabilistic simulation as a means of incorporating stochastic and parametric uncertainty into variables within the model, and propagating these uncertainties along the causal chain to the results, allow the assimilation of various input uncertainties at each step of the modelling process (Aertgeerts and Angelakis, 2003). Uncertainty in results can then be apportioned to each uncertain input variable through the use of rank correlation and importance analysis: knowing where the major uncertainties lie in the assessment model provides useful information on the areas in which additional research or investment might be 
warranted. The full value of using probabilistic approaches was not exploited in some studies that characterised exposure with probability distribution functions, since PFs were still defined deterministically, in spite of the fact that they may represent a major source of uncertainty in an LECR assessment. Bootstrapping was used in exposure assessment in two studies as a means of inferring robust non-parametric probability distribution functions from limited sampling data (Sadiq et al., 2002; Sofuoglu et al., 2003). One method proposed for assessing effects on health of DBPs lacking detailed toxicity data is fuzzy synthetic evaluation (Sadiq and Rodriguez, 2004). Fuzzy sets can be parameterised using weights derived from the carcinogenicity potential ranking system, and arbitrary weights can then be assigned to these sets when they are 'defuzzified'. The advantages of this approach are that it enables a synthesis of cancer and non-cancer risks in one framework; where data are available only in non-commensurate units, vagueness in definitions can be propagated through a causal framework; and the modular form of the methodology is fully scalable to any number of possible contaminants, and health effects etc. The chief limitation of such an approach is that results are highly sensitive to the selection of weights and aggregation operators, which can usually be derived only by using expert opinion.

Population health impact assessment cannot always be a precise science. In producing a scientific answer to a real-world policy problem relating to DBPs, it is usual to make several assumptions, use data of different levels of precision, and employ methods subject to numerous caveats and limitations. These should be recorded, alternatives explored, and results presented in the context of their limitations. In general, it is worthwhile considering the degree of uncertainty of each component of the risk modelling exercise, and assessing whether these are proportional to one another. There is little to be gained from producing very precise exposure estimates if the resolution of that data is later clouded by the much poorer precision of the risk characterisation data or methods, for example.

\subsection{Interpretation and presentation of assessment results - risk communication}

Given the current lack of conclusive evidence of causation of health outcomes among humans exposed to DBPs, any assessment of health risks should be presented alongside a clear acknowledgement of the degree to which results depend on the assumption of causation; such information was largely absent from the vast majority of papers reviewed.

Several studies purported to estimate the cases of cancer attributable to exposure to DBPs, when in fact these estimates are characterised by large uncertainties, and determined partly by embedded value judgements that constitute a part of the regulatory risk framework-based methods and data used. In some cases, policy recommendations made by these studies are disproportionate with the results when viewed in the context the methods used. For example, some studies recommended the substitution of current disinfection treatments (chlorination) with alternatives (e.g. ozonation, chloramination) even when the number of excess cancer cases was negligible over the 70-year lifetime for which they were estimated (Aslan and Turkman, 2007; Chowdhury and Champagne, 2009). In such studies, the potential societal or economic cost implications of such changes were seldom considered, in particular in terms of the potential risks associated with alternative treatments, including other DBPs, for example.

Maps of cancer risk were presented in three studies (Karim et al., 2013; Legay et al., 2011; Venkataraman and Uddameri, 2012) and several of these were based on interpolation, using models that were very weakly supported by data. From a risk communication perspective, presenting high resolution maps of cancer risk runs the danger of masking the much lower resolution and precision of exposure data and ERFs used; the public may imagine that interpolated levels of exposure are reliable and representative of their true state of risk. Apparent spatial inequalities may be little more than artefacts of the overall modelling process.
It is crucial that adequate care is taken in the presentation of results of potentially sensitive assessments. One attributable cancer risk assessment reported that the "total cancer risk analysis indicates that Izmir residents could get cancer from the daily intake of water" (Aslan and Turkman, 2007). Such pronouncements are not helpful for policymakers and could grossly mislead the public and decision-makers. In the context of developing countries where the threat of waterborne disease is particularly serious, presenting what appears to be a scientifically irrefutable claim of disinfection as the cause of cancer may result in unfounded public alarm and bad public health decisions. Ideally, results should be presented in the context of the many uncertainties that characterise them, with recognition of the assumptions that were made to facilitate the assessment, and with due consideration of other risks associated with any recommendations made.

\section{Conclusions}

We reviewed 40 published studies presenting estimates of the public health impacts associated with DBPs in drinking water. With a few notable exceptions, all assessments had weaknesses which we believe seriously reduce the reliability and utility of their results. Firstly, the majority of these studies used upper bound cancer potency factors derived from animal studies to estimate generic lifetime cancer risk or cancer cases attributable to exposure to THMs, the most commonly monitored DBPs, in a particular region or city. Regulatory risk assessment methods and exposure-response data designed for these purposes are intended to overestimate risks (often by an unknown margin) so as to be protective of public health. As such, we consider them inappropriate for accurately predicting realistic levels of risk in target populations. In addition, the lack of specificity of the health outcomes predicted by such methods greatly limits their usefulness in decision-making. We would consider the use of epidemiological data derived from large, well-conducted studies to provide much more accurate, robust and scientifically rigorous estimates of risk in target populations. Many studies were limited by only the most superficial considerations of the true complexity of the occurrence, physicochemical properties and potential relative toxicity of the vast array of chemicals comprising the DBP mixture present in typical chlorinated tap water samples. Additionally, the benefits attributable to disinfection (in terms of reduction of microbial disease) were not considered in the vast majority of studies. Poor characterisation of exposure in the target population, little consideration of the temporally heterogeneous character of that exposure, and a lack of specificity in terms of health outcomes and population characteristics were identified in many of the studies reviewed. Focusing on specific DBPs in the absence of a mechanistic explanation or a true putative agent in the DBP mixture may result in inappropriate or expensive decisions being made in favour of alternative disinfection treatments that may present other health risks, a point convincingly made by Bull (2012).

In this paper we have argued that the use of inappropriate data that inflate risks should be avoided. Only through considered and transparent use of appropriate data, modelling and assumptions, can any assessment of health impacts relating to DBPs be expected to provide useful information to support policy in this area. To our mind, the population health impact assessment should be, above all, a transparent and stringent scientific exercise carried out to provide the best possible answer to a relevant policy question or risk management decision. This might consider any aspect of the system of relevance to the policy, such as possible health effects of alternative water sources, treatment methods or behaviours relating to drinking water, and should consider these in terms of other resulting changes in competing risks such as microbial contamination. The perception that population health impact assessment can be carried out as a simple add-on to an exposure assessment study greatly limits the usefulness of the assessment results. Currently, we consider that, with very few exceptions, existing studies add very little to our understanding of public health implications of DBP exposure, 
and their results could unnecessarily cause alarm among the public which might lead to poor decisions being made by policy-makers and drinking water providers. Future studies should focus on answering real-world policy questions regarding the quality of drinking water, including consideration of both microbial and chemical parameters. They should use up-to-date, appropriate and robust data and methods to answer these questions, and be transparent in explaining the various uncertainties that affect their results, including their quantification where appropriate. While we recognise that the tremendous effort and resources employed by USEPA (2005) in conducting an economic assessment are not typically available to small groups of researchers working in this field, many of the principles underlying that approach can and should be adopted in all health impact assessments relating to DBPs.

\section{Appendix A. Supplementary data}

Supplementary data to this article can be found online at http://dx. doi.org/10.1016/j.envint.2015.02.003.

\section{References}

Aertgeerts, R., Angelakis, A., 2003. State of the Art Report - Health Risks in Aquifer Recharge Using Reclaimed Water. WHO Regional Office for Europe, Copenhagen, Denmark Available at: http://www.who.int/water_sanitation_health/wastewater/wsh0308/en/.

Amjad, H., et al., 2013. Cancer and non-cancer risk assessment of trihalomethanes in urban drinking water supplies of Pakistan. Ecotoxicol. Environ. Saf. 91, 25-31. http://dx.doi.org/10.1016/j.ecoenv.2013.01.008 (PMID: 23453349).

Amy, G.L., Bull, R.J., Craun, G.F., 2000. Environmental Health Criteria 216: Disinfectants and Disinfectant By-products. World Health Organization, Geneva, Switzerland.

Anderson, E.L., USEPA Carcinogen Assessment Group, 1983. Quantitative approaches in use to assess cancer risk. Risk Anal. 3 (4), 277-295. http://dx.doi.org/10.1111/j. 1539-6924.1983.tb01396.x.

Aslan, S., Turkman, A., 2007. In: Mothersill, C., Mosse, I., Seymour, C. (Eds.), Multiple Stressors: A Challenge for the Future. Springer Netherlands, Dordrecht. ISBN: 9781-4020-6335-0.

Attias, L., et al., 1995. Trihalomethanes in drinking water and cancer: risk assessment and integrated evaluation of available data, in animals and humans. Sci. Total Environ. 171 (1-3), 61-68. http://dx.doi.org/10.1016/0048-9697(95)04669-9 (PMID:7481754).

Basu, M., et al., 2011. Multi-route risk assessment from trihalomethanes in drinking water supplies. Environ. Monit. Assess. 178 (1-4), 121-134. http://dx.doi.org/10.1007 s10661-010-1677-z (PMID: 20824332).

Baytak, D., et al., 2008. Seasonal variation in drinking water concentrations of disinfection by-products in Izmir and associated human health risks. Sci. Total Environ. 407 (1), 286-296. http://dx.doi.org/10.1016/j.scitotenv.2008.08.019 (PMID: 18805568).

Bellar, T.A., Lichtenberg, J.J., Kroner, R.C., 1974. The occurrence of organohalides in chlorinated drinking water. J. Am. Water Works Assn. 66, 703-706.

Black, B.D., Harrington, G.W., Singer, P.C., 1996. Reducing cancer risks by improving organic carbon removal. J. Am. Water Works Assn. 88, 40-52 (June).

Briggs, D.J., 2008. A framework for integrated environmental health impact assessment of systemic risks. Environ. Health 7 (1), 61. http://dx.doi.org/10.1186/1476-069X-7-61 (PMID: 19038020).

Bull, R.J., 2012. Toxicological evaluation of experimental data that informs the magnitude of cancer risk from disinfection by-products. Chapter 10. 179-212. In: Hrudey, S.E., Charrois, J.W.A. (Eds.), Disinfection By-products and Human Health. IWA Publishing, London (May).

Bull, R.J., Kopfler, F.C., 1991. Health effects of disinfectants and disinfection by-products. AWWA Res. Fdn, Denver, Colorado.

Bull, R.J., et al., 1995. Water chlorination: essential process or cancer hazard? Fundam. Appl. Toxicol. 28 (2), 155-166 PMID: 8835225.

Bull, R.J., Cotruvo, J.A., Fawell, J., Hrudey, S.E., 2012. Re: Chowdhury et al. 2011. J. Hazard. Mater. Disinfection byproducts in Canadian provinces: associated cancer risks and associated medical expenses. 187: 574-584. J. Hazard. Mater. 237-238, 382-383. http://dx. doi.org/10.1016/j.jhazmat.2011.07.040 (author reply 384-5. PMID: 21974849).

Buteau, S., Valcke, M., 2010. Probabilistic human health risk assessment for quarterly exposure to high chloroform concentrations in drinking-water distribution network of the Province of Quebec, Canada. J. Toxicol. Environ. Health A 73 (23), 1626-1644. http://dx.doi.org/10.1080/15287394.2010.501718 (PMID: 20967676).

Butterworth, B.E., 2005. Science-based risk assessments for drinking water disinfection by-products. Environ. Res. 98 (2), 276-278 (PMID: 15820735)

Butterworth, B.E., Bogdanffy, M.S., 1999. A comprehensive approach for integration of toxicity and cancer risk assessments. Regul. Toxicol. Pharmacol. 29 (1), 23-36 (PMID: 10051416)

Cantor, K.P., 1997. Drinking water and cancer. Cancer Cause Control 8 (3), 292-308 (PMID: PMC1518976)

Chen, W.J., Weisel, C.P., 1998. Halogenated DBP concentrations in a distribution system. J. Am. Water Works Assoc. 90 (4), 151-163.

Chowdhury, S.H., 2012. Response to HAZMAT-D-11-01781 (Ref: Chowdhury S., Rodriguez M. and Sadiq R., 2011. Disinfection byproducts in the Canadian Provinces: associated cancer risks and medical expenses. J Hazard Mater, 187: 574-584). J. Hazard. Mater. 237-238, 384-385. http://dx.doi.org/10.1016/j.jhazmat.2011.09.047.

Chowdhury, S.H., Champagne, P., 2009. Risk from exposure to trihalomethanes during shower: probabilistic assessment and control. Sci. Total Environ. 407 (5), 1570-1578. http://dx.doi.org/10.1016/j.scitotenv.2008.11.025 (PMID: 19131092).

Chowdhury, S., Hall, K., 2010. Human health risk assessment from exposure to trihalomethanes in Canadian cities (retracted). Environ. Int. 36 (5), 453-460. http://dx doi.org/10.1016/j.envint.2010.04.001 (PMID: 20434775).

Chowdhury, S., Rodriguez, M.J., Sadiq, R., 2011. Disinfection byproducts in Canadian provinces: associated cancer risks and medical expenses. J. Hazard. Mater. 187 (1-3), 574-584. http://dx.doi.org/10.1016/j.jhazmat.2011.01.085 (PMID: 21292392).

Chrostowski, P.C., 1994. Exposure assessment principles. In: Patrick, D. (Ed.), Toxic Air Pollution Handbook. Van Nostrand Reinhold, New York, USA, p. 154.

Chung, Y., et al., 1997. Risk assessment and management of drinking water pollutants in Korea. Water Sci. Technol. 36 (12), 309-323. http://dx.doi.org/10.1016/S02731223(97)00734-8.

Coffin, J.C., et al., 2000. Effect of trihalomethanes on cell proliferation and DNA methylation in female B6C3F1 mouse liver. Toxicol. Sci. 58 (2), 243-252 (PMID: 11099637).

Costet, N., et al., 2011. Water disinfection by-products and bladder cancer: is there a European specificity? A pooled and meta-analysis of European case-control studies Occup. Environ. Med. 68 (5), 379-385. http://dx.doi.org/10.1136/oem.2010.062703 (PMID: 21389011).

Crump, K.S., 1984. An improved procedure for low-dose carcinogenic risk assessment from animal data. J. Environ. Pathol. Toxicol. 5 (4-5), 339 (PMID: 6520736).

European Commission, 1998. Council Directive 98/83/EC of 3 November 1998 on the quality of water intended for human consumption. Off. J. Eur. Communities 330/32-330/54.

Fehr, R., et al., 2003. Towards health impact assessment of drinking-water privatization-the example of waterborne carcinogens in North Rhine-Westphalia (Germany). Bull. World Health Organ. 81 (6), 408-414 (PMID: 12894324).

Felter, S., Dourson, M., 1998. The inexact science of risk assessment (and implications for risk management). Hum. Ecol. Risk. Assess. 4 (2), 245-251. http://dx.doi.org/10. 1080/10807039891284316.

Frey, H.C., 1992. Quantitative analysis of uncertainty and variability in environmental policy making. Available at:, http://www4.ncsu.edu/ frey/reports/frey_92.pdf [Accessed July 14,2010$]$.

Gan, W., et al., 2013. The occurrence of disinfection by-products in municipal drinking water in China's Pearl River Delta and a multipathway cancer risk assessment. Sci. Total Environ. 447, 108-115. http://dx.doi.org/10.1016/j.scitotenv.2012.12.091 (PMID: 23376522).

Gratt, L.B., 1996. Air Toxic Risk Assessment and Management: Public Health Risks. John Wiley \& Sons, New York, USA.

Grellier, J., et al., 2010. Exposure to disinfection by-products, fetal growth, and prematurity: a systematic review and meta-analysis. Epidemiology 21 (3), 300-313. http://dx.doi.org/ 10.1097/EDE.0b013e3181d61ffd (PMID: 20375841).

Havelaar, A.H., et al., 2000. Balancing the risks and benefits of drinking water disinfection: disability adjusted life-years on the scale. Environ. Health Perspect. 108 (4), 315-321 (PMID: 10753089).

Hrudey, S.E., 2009. Chlorination disinfection by-products, public health risk tradeoffs and me. Water Res. 43 (8), 2057-2092.

Hsu, C.H., et al., 2001. Estimation of potential lifetime cancer risks for trihalomethanes from consuming chlorinated drinking water in Taiwan. Environ. Res. 85 (2), 77-82 (PMID: 11161657).

Jardine, C.G., Hrudey, S.E., Shortreed, J.H., Craig, L., Krewski, D., Furgal, C., McColl, S., 2003. Risk management frameworks for human health and environmental risks. J. Toxicol. Environ. Health 6, 569-641. http://dx.doi.org/10.1080/10937400390208608.

Jo, W.K., Weisel, C.P., Lioy, P.J., 1990. Chloroform exposure and the health risk associated with multiple uses of chlorinated tap water. Risk Anal. 10 (4), 581-585 (PMID: 2287785).

Karim, Z., Qureshi, B.A., Ghouri, I., 2013. Spatial analysis of human health risk associated with trihalomethanes in drinking water: a case study of Karachi, Pakistan. J. Chem. 1-7 http://dx.doi.org/10.1155/2962.

Kavcar, P., et al., 2006. Occurrence, oral exposure and risk assessment of volatile organic compounds in drinking water for Izmir. Water Res. 40 (17), 3219-3230. http://dx. doi.org/10.1016/j.watres.2006.07.002 (PMID: 16928390).

King, B., 2010. Personal Communication.

Lahey, W., Connor, M., 1983. The case for ocean waste-disposal. Technol. Rev. 86 (6), 60-68.

LaKind, J.S., et al., 2010. Public health interpretation of trihalomethane blood levels in the United States: NHANES 1999-2004. J. Expo. Sci. Environ. Epidemiol. 20 (3), 255-262. http://dx.doi.org/10.1038/jes.2009.35 (PMID: 19550438).

Lee, S.C., et al., 2004. Multipathway risk assessment on disinfection by-products of drinking water in Hong Kong. Environ. Res. 94 (1), 47-56 (PMID: 14643286).

Lee, J., et al., 2013. Occurrence of disinfection by-products in tap water distribution systems and their associated health risk. Environ. Monit. Assess. 185 (9), 7675-7691. http://dx.doi.org/10.1007/s10661-013-3127-1 (PMID: 23446885).

Legay, C., et al., 2011. Spatial variations of human health risk associated with exposure to chlorination by-products occurring in drinking water. J. Environ. Manage. 92 (3), 892-901. http://dx.doi.org/10.1016/j.jenvman.2010.10.056 (PMID: 21093977).

Liu, S., et al., 2011. Seasonal variation effects on the formation of trihalomethane during chlorination of water from Yangtze River and associated cancer risk assessment. J. Environ. Sci. 23 (9), 1503-1511. http://dx.doi.org/10.1016/S1001-0742(10)60573-6 (PMID: 22432287)

Malcolm, M.S., Weinstein, P., Woodward, A.J. 1999. Something in the water? A health impact assessment of disinfection by-products in New Zealand. N. Z. Med. J. 6 (3), 1-2 (PMID: 10606403). 
Morris, R.D., et al., 1992. Chlorination, chlorination by-products, and cancer: a metaanalysis. Am. J. Public Health 82 (7), 955-964 (PMID: 1535181).

Navarro, I., et al., 2007. Assessment of potential cancer risks from trihalomethanes in water supply at mexican rural communities. International Symposium on New Directions in Urban Water Management. UNESCO, Paris, pp. 1-8.

Nazir, M., Khan, F.I., 2006. Human health risk modeling for various exposure routes of trihalomethanes (THMs) in potable water supply. Environ. Model Softw. 21 (10), 1416-1429. http://dx.doi.org/10.1016/j.envsoft.2005.06.009 (PMID:)

Nieuwenhuijsen, M.J., Toledano, M.B., Eaton, N.E., Fawell, J., Elliott, P., 2000a. Chlorination disinfection byproducts in water and their association with adverse reproductive outcomes: a review. Occup. Environ. Med. 57 (2), 73-85. http://dx.doi.org/10.1136/oem. 57.2.73 (PMID: 10711274).

Nieuwenhuijsen, M.J., Toledano, M.B., Elliott, P., 2000b. Uptake of chlorination disinfection by-products; a review and a discussion of its implications for exposure assessment in epidemiological studies. J. Expo. Anal. Environ. Epidemiol. 10 (6 Pt 1), 586-599. http://dx.doi.org/10.1038/sj.jea.7500139 (PMID: 11140442).

Nieuwenhuijsen, M.J., et al., 2009a. The epidemiology and possible mechanisms of disinfection by-products in drinking water. Phil. Trans. R. Soc. A 367 (1904), 4043-4076. http:// dx.doi.org/10.1098/rsta.2009.0116 (PMID: 19736233).

Nieuwenhuijsen, M.J., et al., 2009b. Chlorination disinfection by-products in drinking water and congenital anomalies: review and meta-analyses. Environ. Health Perspect. 117 (10), 1486-1493. http://dx.doi.org/10.1289/ehp.0900677 (PMID: 20019896).

Nieuwenhuijsen, M.J., et al., 2010. Literature review of meta-analyses and pooled analyses of disinfection by-products in drinking water and cancer and reproductive health outcomes. In: Haldane, R. (Ed.), Contaminants of Emerging Concern in the Environment: Ecological and Human Health Considerations. ACS Publications, Washington, DC, pp. 482-496 http://dx.doi.org/10.1021/bk-2010-1048.ch024.

Nieuwenhuijsen, M.J., et al., 2013. Environmental risk factors of pregnancy outcomes: a summary of recent meta-analyses of epidemiological studies. Environ. Health 12 (1), 6. http://dx.doi.org/10.1186/1476-069X-12-6 (PMID: 23320899).

NTP, 2005. Report on carcinogens. http://ntp.niehs.nih.gov/ntp/roc/toc11.html.

Pardakhti, A.R., et al., 2011. Comparative cancer risk assessment of THMs in drinking water from well water sources and surface water sources. Environ. Monit. Assess. 179 (1-4), 499-507. http://dx.doi.org/10.1007/s10661-010-1752-5 (PMID: 20981567)

Parvez, S., et al., 2011. Temporal variability in trihalomethane and haloacetic acid concentrations in Massachusetts public drinking water systems. Environ. Res. 111 (4), 499-509. http://dx.doi.org/10.1016/j.envres.2010.12.008 (PMID: 21316653).

Plewa, M.J., Wagner, E.D., 2009. Mammalian Cell Cytotoxicity and Genotoxicity of Disinfection By-products. Water Research Foundation, Denver, Colorado, US

Plewa, M.J., et al., 2004. Chemical and biological characterization of newly discovered iodoacid drinking water disinfection byproducts. Environ. Sci. Technol. 38 (18), 4713-4722. http://dx.doi.org/10.1021/es049971v (PMID: 15487777).

Plewa, M.J., et al., 2008. Occurrence, synthesis, and mammalian cell cytotoxicity and genotoxicity of haloacetamides: an emerging class of nitrogenous drinking water disinfection byproducts. Environ. Sci. Technol. 42 (3), 955-961. http://dx.doi.org/10. 1021/es071754h (PMID: 18323128).

Pontius, F., 2003. Drinking Water Regulation and Health. Wiley, IEEE.

Reckhow, K.H., 1994. Importance of scientific uncertainty in decision making. Environ. Manag. 18 (2), 161-166. http://dx.doi.org/10.1007/BF02393758.

Richardson, S.D., 1998. Drinking Water Disinfection By-products. Encyclopedia Environmental Analysis and Remediation 3, pp. 1398-1421.

Richardson, S.D., 2005. New disinfection by-product issues: emerging DBPs and alternative routes of exposure. Global NEST J. 7 (1), 43-60.

Richardson, S.D., et al., 2007. Occurrence, genotoxicity, and carcinogenicity of regulated and emerging disinfection by-products in drinking water: a review and roadmap for research. Mutat. Res. 636 (1-3), 178-242 (PMID: 17980649).

Rodricks, J.V., 2007. 2006 American College of Toxicology distinguished service award lec ture: has quantitative risk assessment been of benefit to the science of toxicology. Int J. Toxicol. 26, 3-12 (PMID: 17365141).

Rodriguez, M.J., Serodes, J.B., 2001. Spatial and temporal evolution of trihalomethanes in three water distribution systems. Water Res. 35 (6), 1572-1586. http://dx.doi.org/ 10.1016/S0043-1354(00)00403-6 (PMID: 11317905).

Rook, J.J., 1974. Formation of haloforms during chlorination of natural waters. Water Treat. Exam. 23, 234-243.

Sadiq, R., Rodriguez, M.J., 2004. Disinfection by-products (DBPs) in drinking water and predictive models for their occurrence: a review. Sci. Total Environ. 321 (1-3), 21-46. http://dx.doi.org/10.1016/j.scitotenv.2003.05.001.

Sadiq, R., Husain, T., Kar, S., 2002. Chloroform associated health risk assessment using bootstrapping: a case study for limited drinking water samples. Water Air Soil Pollut 138 (1-4), 123-140. http://dx.doi.org/10.1023/A:1015579722245.

Sander, P., Bergbäck, B., Oberg, T., 2006. Uncertain numbers and uncertainty in the selection of input distributions-consequences for a probabilistic risk assessment of contaminated land. Risk Anal. 26 (5), 1363-1375. http://dx.doi.org/10.1111/j.1539-6924.2006.00808.x (PMID: 17054537).

Semerjian, L., Dennis, J., 2007. Multipathway risk assessment of trihalomethane exposure in drinking water of Lebanon. J. Water Health 5 (4), 511-522 (PMID: 17878564).

Smith, R.L., 1996. Risk-based concentrations: prioritizing environmental problems using limited data. Toxicology 106 (1-3), 243-266. http://dx.doi.org/10.1016/0300483X(95)03165-C (PMID:8571397).

Smith, V.L., et al., 1980. Temporal variations in trihalomethane content of drinking water. Environ. Sci. Technol. 14 (2), 190-196. http://dx.doi.org/10.1021/es60162a008.

Sofuoglu, S.C., et al., 2003. Exposure and risk estimates for Arizona drinking water. J. Am. Water Works Assoc. 95 (7), 67-79. http://dx.doi.org/10.1016/j.ijheh. 2008.05.002.

Tokmak, B., et al., 2004. Trihalomethanes and associated potential cancer risks in the water supply in Ankara, Turkey. Environ. Res. 96 (3), 345-352. http://dx.doi.org/10. 1016/j.envres.2003.11.005 (PMID: 15364603).

Toledano, M.B., et al., 2005. Relation of trihalomethane concentrations in public water supplies to stillbirth and birth weight in three water regions in England. Environ. Health Perspect. 113 (2), 225-232. http://dx.doi.org/10.1289/ehp.7111 (PMID: 15687062).

Tuomisto, J., 2005. Does mechanistic understanding help in risk assessment-the example of dioxins. Toxicol. Appl. Pharmacol. 207 (2 Suppl.), 2-10. http://dx.doi.org/10.1016/j. taap.2005.01.053 (PMID: 15996698).

USEPA, 1986. Guidelines for Carcinogen Risk Assessment (EPA/600/8-87/045).

USEPA, 1994. National primary drinking water regulations: disinfectants/disinfection byproducts. Proposed rule. Fed. Regist. 59 (145), 38667.

USEPA, 1998. National primary drinking water regulations: disinfectants and disinfection byproducts. Final rule. Fed. Regist. 63 (241), 69406-69407.

USEPA, 1999. Guidelines for Carcinogen Risk Assessment (NCEA-F-0644 (revised draft)).

USEPA, 2002a. IRIS Integrated Risk Information System (Electronic database). Available at:. http://www.epa.gov/iris.

USEPA, 2002b. Guidelines for Carcinogen Risk Assessment. Risk Assessment Forum (NCEA-F-0644 (revised draft))

USEPA, 2004. EPA/540/R/99/005 Risk Assessment Guidance for Superfund Volume I: Human Health Evaluation Manual (Part E, Supplemental Guidance for Dermal Risk Assessment). USEPA, Washington, DC.

USEPA, 2005. Economic Analysis for the Final Stage 2 Disinfectants and Disinfection Byproducts Rule. EPA, Washington, DC (815-R-05-010).

USEPA, 2013. Basic information about disinfection byproducts in drinking water: total trihalomethanes, haloacetic acids, bromate, and chlorite. Available at:, http://water.epa. gov/drink/contaminants/basicinformation/disinfectionbyproducts.cfm\# (What disinfection byproducts does EPA regulate, how are they formed, and what are their health effects in drinking water at levels above the maximum contaminant level?, Accessed April 14, 2014).

Uyak, V., 2006. Multi-pathway risk assessment of trihalomethanes exposure in Istanbul drinking water supplies. Environ. Int. 32 (1), 12-21. http://dx.doi.org/10.1016/j. envint.2005.03.005 (PMID: 16154195)

Venkataraman, K., Uddameri, V., 2012. A GIS-based evaluation of risks due to trihalomethane exposure during showering in coastal Texas. Clean Technol. Environ. 14 (4), 551-564. http://dx.doi.org/10.1007/s10098-011-0415-8.

Viana, R.B., et al., 2009. Risk assessment of trihalomethanes from tap water in Fortaleza, Brazil. Environ. Monit. Assess. 151 (1), 317-325. http://dx.doi.org/10.1007/s10661008-0273-y (PMID: 18365760).

Villanueva, C.M., et al., 2003. Meta-analysis of studies on individual consumption of chlorinated drinking water and bladder cancer. J. Epidemiol. Commun. Health 57 (3), 166-173. http://dx.doi.org/10.1136/jech.57.3.166 (PMID: 12594192).

Villanueva, C.M., et al., 2004. Disinfection byproducts and bladder cancer: a pooled analysis. Epidemiology 15 (3), 357-367. http://dx.doi.org/10.1097/01.ede.0000121380. 02594.fc (PMID: 15097021).

Wang G.S., Deng Y.C. Lin, T.F. 2007a Cancer risk assessment from trihalomethanes in drinking water. Sci. Total Environ. 387 (1-3), 86-95. http://dx.doi.org/10.1016/j. scitotenv.2007.07.029 (PMID: 17727920)

Wang, W., et al., 2007b. Risk assessment on disinfection by-products of drinking water of different water sources and disinfection processes. Environ. Int. 33 (2), 219-225. http://dx.doi.org/10.1016/j.envint.2006.09.009 (PMID: 17056115).

WHO, 2006. Guidelines for drinking-water quality, 3rd ed. Vol. 1. Recommendation. Available at:. http://whqlibdoc.who.int/publications/2004/9241546387.pdf.

Williams, P., et al., 2002. Comparative risk analysis of six volatile organic compounds in California drinking water. Environ. Sci. Technol. 36 (22), 4721-4728. http://dx.doi. org/10.1021/es020725y (PMID: 12487291).

Yamamoto, K., 2011. Is advanced treated water truly high quality?: the answer from a viewpoint of cancer risk induced by trihalomethane class. Bull. Environ. Contam. Toxicol. 87 (2), 101-105. http://dx.doi.org/10.1007/s00128-011-0321-0 (PMID: 21660527) 